\title{
Retraction \\ Retracted: Influence of L-Tryptophan on Growth and Optical Properties of PbS Nanocrystalline Thin Films
}

\section{Journal of Nanomaterials}

Received 16 December 2020; Accepted 16 December 2020; Published 26 February 2021

Copyright (c) 2021 Journal of Nanomaterials. This is an open access article distributed under the Creative Commons Attribution License, which permits unrestricted use, distribution, and reproduction in any medium, provided the original work is properly cited.

Journal of Nanomaterials has retracted the article titled "Influence of L-Tryptophan on Growth and Optical Properties of PbS Nanocrystalline Thin Films" [1]. As originally raised on PubPeer [2], the article was found to have concerns with some of the figures. A summary of the concerns is as follows:

(i) Figures 4(a), 4(c), and 4(e) (left-hand side) are identical to Figures 2(a), 2(b), and 2(c) in [3] despite [1] using tryptophan and [3] using lithium. A corrigendum has since been published to replace these figures in the other article [4].

(ii) Figure 4(a), 4(c), and 4(e) (right-hand side) are identical to Figures 1(a), 1(b), and 1(c) in [5], despite [1] using tryptophan and [5] using bismuth.

(iii) In Figure 14(a), the absorption spectra for PbS-T5 and PbS-T10 appear to be identical.

(iv) In Figure 3, the PbS-T30 and PbST20 traces appear to be identical. The PbS spectrum (black) also appears to be identical with the PbS spectrum in [6].

The journal and the editorial board are retracting the article due to concerns that the data in this article are not reliable. The authors do not agree to retraction.

\section{References}

[1] R. Palomino Merino, R. Gutiérrez Pérez, P. Trejo García et al., "Influence of L-Tryptophan on Growth and Optical Properties of PbS Nanocrystalline Thin Films," Journal of Nanomaterials, vol. 2018, Article ID 3431942, 15 pages, 2018.
[2] "Influence of L-Tryptophan on Growth and Optical Properties of PbS Nanocrystalline Thin Films," PubPeer, 2019, https:// pubpeer.com/publications/8D16E32378DE46D37140B5FC 5EA575.

[3] M. Chávez Portillo, J. Alvarado Pulido, S. Gallardo Hernández et al., "Voc enhancement of a solar cell with doped $\mathrm{Li}^{+}-\mathrm{PbS}$ as the active layer," Superlattices and Microstructures, vol. 118, pp. 137-144, 2018.

[4] M. Chávez Portillo, J. Alvarado Pulido, S. Gallardo Hernández et al., "Corrigendum to "Voc enhancement of a solar cell with doped $\mathrm{Li}+-\mathrm{PbS}$ as the active layer"," Superlattices and Microstructures, vol. 138, 2020.

[5] R. Gutiérrez Pérez, O. Portillo Moreno, L. Chaltel Lima, R. Palomino Merino, and M. Zamora Totozintle, "Optical and structural properties of PbS: $\mathrm{Bi}^{3+}$ nanocrystals," Revista Mexicana de Física, vol. 61, pp. 356-362, 2015, https:// scholar.google.com/scholar?hl=en\&as_sdt=0\%2C5\&q=optical + and + structural + properties + of + pbs $\% 3 \mathrm{~A}+\mathrm{bi} 3 \% 2 \mathrm{~B}$ + nanocrystals $+\& b \operatorname{btnG}=$.

[6] O. Portillo-Moreno, R. Gutierrez-Perez, M. Chavez Portillo et al., "Growth of doped PbS: $\mathrm{Co}^{2+}$ nanocrystals by Chemical Bath," Revista Mexicana de Fisica, vol. 62, pp. 456-460, 2016. 


\title{
Influence of L-Tryptophan on Growth and Optical Properties of PbS Nanocrystalline Thin Films
}

\author{
R. Palomino Merino, ${ }^{1}$ R. Gutiérrez Pérez, ${ }^{2}$ P. Trejo García ${ }^{D},{ }^{1}$ L. Chaltel Lima, ${ }^{2}$ \\ O. Portillo Moreno, ${ }^{2}$ M. E. Araiza García, ${ }^{3}$ A. Moreno Rodriguez, ${ }^{2}$ and E. Rubio Rosas ${ }^{4}$ \\ ${ }^{1}$ Facultad de Ciencias Físico Matemáticas, Universidad Autónoma de Puebla, 72570 Puebla, PUE, Mexico \\ ${ }^{2}$ Facultad de Ciencias Quimicas, Universidad Autonoma de Puebla, 72592 Puebla, PUE, Mexico \\ ${ }^{3}$ Vía Atlixcayotl 2301 Col. Reserva Territorial. Atlixcayotl, ITESM, Campus Puebla, Puebla, 72001 PUE, Mexico \\ ${ }^{4}$ Prolongación de la 24 Sur y Av. San Claudio, Ciudad Universitaria, Col. San Manuel, CUVyTT, 72570 Puebla, PUE, Mexico
}

Correspondence should be addressed to P. Trejo García; pablo.trejogarcia@alumno.buap.mx

Received 4 April 2018; Accepted 22 May 2018; Published 5 July 2018

Academic Editor: William Yu

Copyright (c) 2018 R. Palomino Merino et al. This is an open access article distributed under the Creative Commons Attribution License, which permits unrestricted use, distribution, and reproduction in any medium, provided the original work is properly cited.

\begin{abstract}
The growth through the green chemical bath of $\mathrm{PbS}$ doped systematically with the biomolecule L-tryptophan led to growth of hybrid, inorganic-organic, nanocrystalline thin films onto glass slides at $\mathrm{T} \sim 80^{\circ} \mathrm{C}$. The thickness was found in the range of 230-140 nm. Morphological changes were analyzed using atomic force microscopy (AFM). FTIR (Fourier-transform infrared spectroscopy) spectra showed broad absorption bands located at $\sim 3450 \mathrm{~cm}^{-1}$ attributed to stretching of the $\mathrm{H}_{2} \mathrm{O}$ molecules and two small absorption bands located at $\sim 2285 \mathrm{~cm}^{-1}$ and $\sim 2918 \mathrm{~cm}^{-1}$ along with a strong band at $\sim 1385 \mathrm{~cm}^{-1}$ assigned to vibration modes corresponding to $\mathrm{CO}_{3}^{2-}$ ions. In the patterns of X-ray diffraction (XRD), the cubic phase was identified in all the samples according to the angular positions $2 \theta \sim 26.08^{\circ}, 30.13^{\circ}, 43.08^{\circ}, 51.91^{\circ}, 53.60^{\circ}, 6251^{\circ}, 68.98^{\circ}$, and $71.15^{\circ}$. Using the Scherrer formula on the XRD patterns, the grain size (GS) was determined; for the undoped sample, $\sim 42 \mathrm{~nm}$ was found, whereas for the doped samples, $\sim 42-22 \mathrm{~nm}$ was found. The electronic charge distribution of L-tryptophan was determined using the molecular electrostatic potential (MEP) to understand the decrease on the GS associated with the interaction of $\pi$ electrons from conjugated rings and amino-acid functional groups. The absorbance spectra in doped films showed excitonic peaks at $\sim 1.8-2.1 \mathrm{eV}$ associated to a higher energy of the ${ }^{1} \mathrm{~S}_{\mathrm{h}} \rightarrow{ }^{1} \mathrm{~S}_{\mathrm{h}}$ and ${ }^{1} \mathrm{P}_{\mathrm{h}} \rightarrow{ }^{1} \mathrm{P}_{\mathrm{e}}$ electronic transitions. Through optical absorption, a shift for the band gap energy was observed from $\sim 1.4 \mathrm{eV}$ for the undoped sample and $\sim 2.1-2.3 \mathrm{eV}$ for the doped films, respectively. Such behaviour is generally associated with the GS decrease and the effect of quantum confinement; a simple model by calculating changes in Gibbs free energy $\left(\Delta \mathrm{G}^{\circ}\right)$ for growth of nanocrystals is presented.
\end{abstract}

\section{Introduction}

$\mathrm{PbS}$ is a direct-band semiconductor with a relatively small band gap energy $\left(\mathrm{E}_{\mathrm{g}}\right) \sim 0.41 \mathrm{eV}$ at $300 \mathrm{~K}$ and can be used for fabrication on mid-infrared detectors, solar energy absorption devices, and optical switches [1]; it has also been thoroughly investigated for its quantum confinement effect in which the wavelength dependency of optical absorption and emission process can be monitored over a wide range of energy [2]. This semiconductor has the advantage of modifying its morphological, structural, optical, and electrical properties by means of systematic doping with different cations and anions. Latest reports of doped-PbS nanocrystals with different monovalent and divalent ions and even with three inorganic ions which have been systematically incorporated in the crystal lattice showed the different reasons for investigating this semiconductor in depth according to the interesting changes observed $[3,4]$. As it is well-known, this semiconductor has been synthesized using various techniques, such as atomic layer deposition [5], SILAR [6], 
hydrothermal synthesis [7], hot injection method [8], and chemical bath deposition [9]. This last technique has been used in the last decades for the synthesis of various semiconductor materials, and as such, the chemical bath (CB) approach is a green, simple, and cost-effective approach in which the material obtained is of good crystalline quality with acceptable stoichiometry and worthy of being applied for $\mathrm{PbS}$ synthesis.

In the doped-PbS material, it is possible to control the crystal size and, consequently, an $\mathrm{E}_{\mathrm{g}}$ shifting in a significant way at different intervals according to the doping technique, temperature, reaction time, stirring, $\mathrm{pH}$, and so on.

$\mathrm{E}_{\mathrm{g}}$ shifts in the range of the spectral region between $\sim 0.4$ and $3.5 \mathrm{eV}$ when the GS decreases below the size of the excitonic Bohr radius $(\sim 18 \mathrm{~nm})$ due to the quantum confinement effect; this is one of the characteristics worthy of investigation in this material. There are several reports that have shown that in doping systematically with different inorganic ions, it is possible to modify the morphological, structural, optical, and electrical properties $[3,4,10]$. Some other reports of doping with metalorganic compounds in $\mathrm{PbS}$ have shown results that attract attention by charge transfer along with structural and optical changes [11], a phenomenon that establishes the importance of doping with different organic molecules. However, doping with organic molecules is still in the preliminary stage since only few reports have been published with CdS [12] and CdO [13].

The biomolecule L-tryptophan $\left(\mathrm{C}_{11} \mathrm{H}_{12} \mathrm{~N}_{2} \mathrm{O}_{2}\right.$ (2S)-2amino-3-(1H-indol-3-yl)propanoic acid) is soluble in water and does not require the use of nonaqueous solvents that are difficult to remove during crystalline growth, so it can be introduced by adding in situ aqueous solution to the growth process of a $\mathrm{PbS}$ matrix. In the present report, the green $\mathrm{CB}$ technique is employed to grow doped $\mathrm{PbS}$ with the biomolecule (L-tryptophan), and the stability of the biomolecule under the temperature of crystal growth of $\sim 80^{\circ} \mathrm{C}$ due to the presence of conjugated double bonds is proposed; a chiral carbon ( $s p^{3}$-hybridized) and amino acid functional group can induce interactions in the crystalline growth of $\mathrm{PbS}$.

\section{Experimental Procedure}

2.1. Chemical Reactions. Reactions for the growth of doped$\mathrm{PbS}$ nanocrystals in a L-tryptophan solution were investigated employing the cell potential $\left(\varepsilon^{\circ}\right)$ already reported [14]. The Nernst equation $\Delta G^{\circ}=-n \tau \varepsilon^{\circ}$ derived from the Gibbs-free energy, where $n$ is the number of equivalents and $\tau$ is the Faraday constant $(96,485 \mathrm{C} / \mathrm{mol})$, was used. The numerical value calculation of $\Delta G^{\circ}$ provides empirical information of the spontaneity of them corresponding chemical reaction, associated with the $(+)$ or $(-)$ sign, respectively. The chemical reactions are presented below.

Hydrolysis of thiourea generates the following ions:

$$
\mathrm{SC}\left(\mathrm{NH}_{2}\right)_{2}+4 \mathrm{OH}^{-} \Leftrightarrow \mathrm{CO}_{3}^{2-}+\mathrm{S}^{2-}+2 \mathrm{NH}_{3}+\mathrm{H}_{2} \mathrm{O} .
$$

The $\mathrm{Pb}^{2+}$ ions are generated in the alkaline medium according to the following dissociation reaction:

$$
\mathrm{Pb}\left(\mathrm{CH}_{3} \mathrm{COO}\right)_{2} \Leftrightarrow \mathrm{Pb}^{2+}+2 \mathrm{CH}_{3} \mathrm{COO}^{-} .
$$

The coordination complex $\left[\mathrm{Pb}\left(\mathrm{NH}_{3}\right)_{4}\right]^{2+}$ ion is generated indirectly by mixing the following reagents $\mathrm{Pb}\left(\mathrm{CH}_{3} \mathrm{COO}\right)_{2}$, $\mathrm{KOH}$, and $\mathrm{NH}_{4} \mathrm{NO}_{3}$. The formation of the coordination complex is key for the release of $\mathrm{Pb}^{2+}$ with $\mathrm{S}^{2-}$ ions under these conditions:

$$
\left[\mathrm{Pb}\left(\mathrm{NH}_{3}\right)_{4}\right]^{2+}+\mathrm{S}^{2-} \Leftrightarrow \mathrm{PbS}+4 \mathrm{NH}_{3} \quad \Delta \mathrm{Go}=+362.8 \mathrm{KJ} .
$$

Therefore, $\Delta G^{\circ}>0$, and as such, the reaction is not a spontaneous process. In the case of tryptophan, the following dissociation is proposed in alkaline medium [15]

$$
\mathrm{R}-\mathrm{COOH}+\mathrm{H}_{2} \mathrm{O} \Leftrightarrow \mathrm{R}-\mathrm{COO}^{-}+\mathrm{H}_{3} \mathrm{O}^{+} \quad \Delta \mathrm{G}^{\circ}=-1949.0 \mathrm{~kJ},
$$

where $\mathrm{R}-\mathrm{COOH}$ is the L-tryptophan solution. Adding (3) and (4),

$$
\begin{gathered}
{\left[\mathrm{Pb}\left(\mathrm{NH}_{3}\right)_{4}\right]^{2+}+\mathrm{S}^{2-}+\text { L-tryptophan } \Leftrightarrow \underbrace{\mathrm{PbS}: \text { L-tryptophan }}_{\text {Nanocrystal }}} \\
+4 \mathrm{NH}_{3} \quad \Delta \mathrm{G}^{\circ}=-1586.2 \mathrm{~kJ} .
\end{gathered}
$$

These semiempirical calculations show that the thermodynamic equilibrium in (5) is a spontaneous process $\left(\Delta G^{\circ}\right.$ $<0$ ) and is thermodynamically probable. On the other hand, L-tryptophan is thermally stable until $\sim 251^{\circ} \mathrm{C}$ in air atmosphere [16]. Thus, decomposition of L-tryptophan is not favourable assuring that this molecule is not fragmented in our working conditions.

2.2. Preparation of the Films. The technique for the film preparation is used in the green CB approach. In the aforementioned synthesis of the $\mathrm{PbS}$ films doped systematically with the biomolecule L-tryptophan solution labelled $\left(\mathrm{V}_{\text {[L-trypto- }}\right.$ phan] $)$, we proceed to prepare the glass substrates, which were previously immersed in a $\mathrm{K}_{2} \mathrm{Cr}_{2} \mathrm{O}_{7}: \mathrm{HCl}: \mathrm{H}_{2} \mathrm{O}$ solution for $\sim 24.0 \mathrm{~h}$, after which they were rinsed in deionised water $(\sim 18.2 \mathrm{M} \Omega)$ and dried in a clean and hot air flow. The growth of PbS films with five levels of doping L-tryptophan solution was obtained by the addition in situ of 5, 10, 15, 20, and $30 \mathrm{~mL}$ in the solutions for $\mathrm{PbS}$ crystal growth. The concentrations of precursor reagents were $\mathrm{Pb}\left(\mathrm{CH}_{3} \mathrm{COO}\right)_{2}(0.01 \mathrm{M})$, $\mathrm{KOH}(0.5 \mathrm{M}), \mathrm{NH}_{4} \mathrm{NO}_{3}(1.5 \mathrm{M})$, and $\mathrm{SC}\left(\mathrm{NH}_{2}\right)_{2}(0.2 \mathrm{M})$. All precursor reagents were $99.9 \%$ pure from Sigma-Aldrich and were used directly. The growing time was $\sim 15.0 \mathrm{~min}$ at constant temperature deposition $\left(\mathrm{T}_{\mathrm{d}}\right) \sim 80^{\circ} \mathrm{C}$. The $\mathrm{pH}$ of the total mixture was quantified in the $~ 8.3-8.4$ range, remaining practically constant. Because the solution was routinely added to the reaction mixture during the crystalline growth of films, the samples were labelled as $\mathrm{PbS}$ for the undoped film and PbS-T5, PbS-T10, PbS-T20, and PbS-T30 for doped samples. The films obtained showed a change in the coloration of metallic black crystals to dark brown color. The FTIR spectrum was recorded using a Perkin Elmer spectrophotometer in the $\sim 500-4000 \mathrm{~cm}^{-1}$ wavelength regions. Layer 


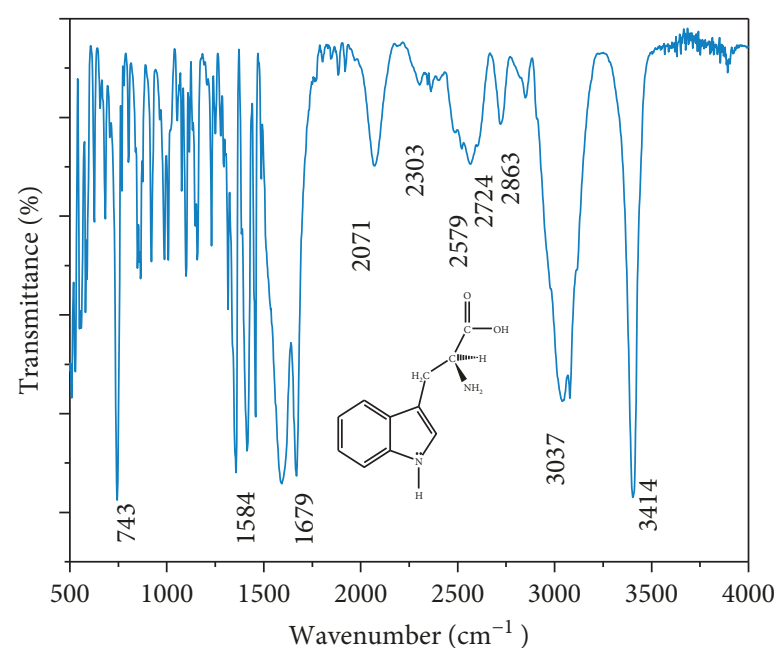

FIGURE 1: FTIR spectrum of the L-tryptophan biomolecule.

thickness was in the $\sim 230-140 \mathrm{~nm}$ range, as determined by utilizing a Dektak II profilometer. The AFM images of the films were registered by a Nanoscope E model contact mode AFM. Crystalline structure characterization was carried out by XRD patterns registered in a D8 Discover diffractometer, using the $\mathrm{CuK} \alpha$ line $\sim 1.4 \AA$. GS was determined utilizing Scherer's formula on XRD patterns. The optical absorption spectra, measured employing a Unicam 8700 spectrometer, allowed calculating the band gap energy by using the $(\alpha \mathrm{h} v)^{2}$ versus h $v$ plot, where $\alpha$ is the optical absorption coefficient and $h v$ is the photon energy.

\section{Results and Discussion}

The FTIR technique was used to investigate the incorporation of L-tryptophan into the PbS matrix. FTIR spectra of PbS-PbS-T films were obtained over a $\sim 500-4000 \mathrm{~cm}^{-1}$ range. The typical FTIR spectra of L-tryptophan [17] are shown in Figure 1, and the structure is shown in the lower part centered in Figure 1. For this biomolecule, an indole group with an alanine side chain can be observed. The total number of atoms is 27 , and hence, the number of vibrational frequencies is 25 located at 51 in-plane vibrations and 24 outof-plane vibrations [18]. The vibrational modes are $>\mathrm{C}-\mathrm{H}$, $>\mathrm{C}=\mathrm{O},-\mathrm{O}-\mathrm{H}$, and $-\mathrm{N}-\mathrm{H}$ (indole) stretch; $-\mathrm{N}-\mathrm{H}$ (amine) and $-\mathrm{C}-\mathrm{C}-$ stretch; and $-\mathrm{NH}_{4}^{+},-\mathrm{COO}^{-}$bending. Therefore, mainly broad bands located at $\sim 1500-3500 \mathrm{~cm}^{-1}$ can be assigned to combined effects of intermolecular and intramolecular hydrogen bonding. The $-\mathrm{COO}^{-}$antisymmetric stretch located at $\sim 1679 \mathrm{~cm}^{-1}$ generally can be related with $\mathrm{H}_{2} \mathrm{~N}-\mathrm{H}$ antisymmetric bending modes at $\sim 1584 \mathrm{~cm}^{-1}$ [15].

Bands located at $\sim 3414 \mathrm{~cm}^{-1}(-\mathrm{N}-\mathrm{H}$ stretching in the amine group) and broad bands at $\sim 3037, \sim 2863, \sim 2579$, and $\sim 2724$ (C-H stretching); $\sim 2303$ and $\sim 2071 \mathrm{~cm}^{-1}(-\mathrm{C}=\mathrm{N}-$ stretching); 1679 and $\sim 1584$ (-C-C- stretching); and $\sim 743 \mathrm{~cm}^{-1}$ can be observed. According to (1), thiourea hydrolysis generates the $\mathrm{CO}_{3}^{2-}$ anion, and in the alkaline reaction medium ( $\mathrm{pH} \sim 8.3-8.4$ ), the $\mathrm{OH}^{-}$ions are found. FTIR spectra of thiourea solution are shown in Figure 2. FTIR spectra of the $\mathrm{PbS}-\mathrm{PbS}-\mathrm{T}$ nanocrystal are shown in Figure 3, and little

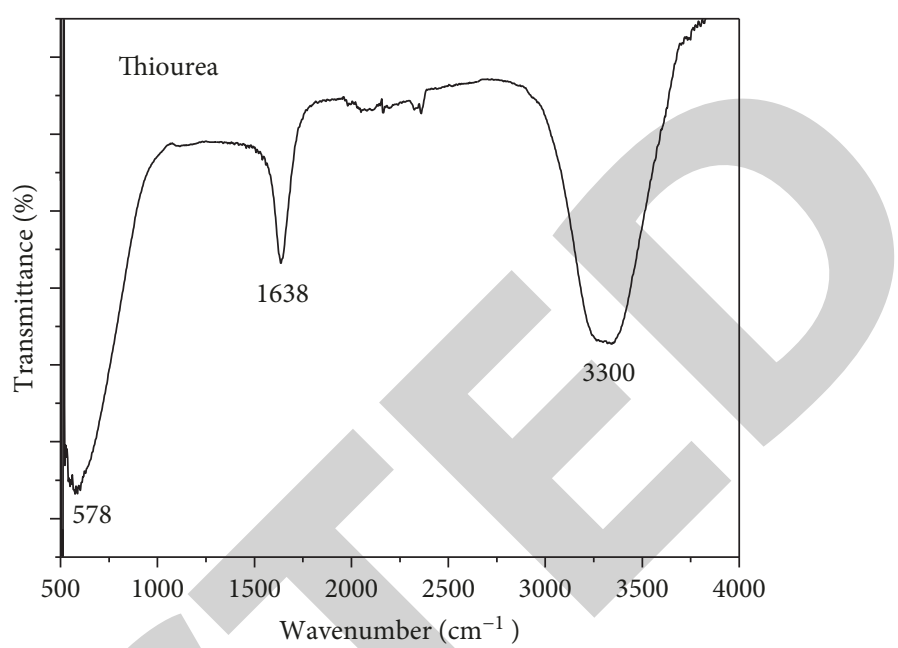

FIGURE 2: FTIR spectrum of thiourea solution. The band identified with the $\mathrm{OH}^{-}$and $\mathrm{CO}_{3}^{2-}$ ions can be seen.

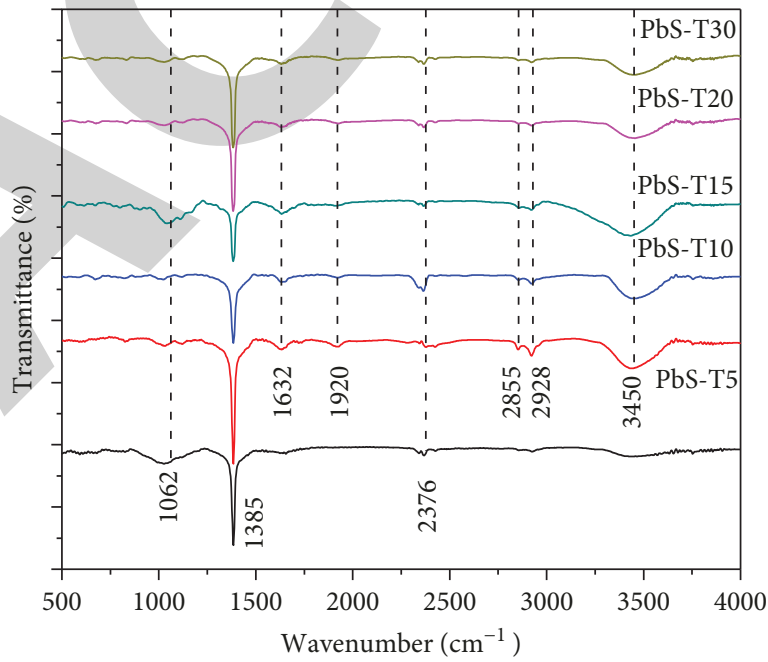

FIgURE 3: FTIR of the PbS-PbS-T nanocrystal. Bands associated with L-tryptophan are not appreciated.

changes can be appreciated when the undoped $\mathrm{PbS}$ nanocrystal is compared with $\mathrm{PbS}-\mathrm{T}$ films. The absorption bands attributed to the vibrations in $\mathrm{CO}_{3}^{2-}$ anions are located in the range of $\sim 400-1600 \mathrm{~cm}^{-1}$ [19].

In this FTIR spectrum, a strong and sharp absorption band is presented in all the nanocrystals located at $\sim 1385 \mathrm{~cm}^{-1}$, differing only in intensity, which is attributed to asymmetric stretching vibration of the $\mathrm{CO}_{3}^{2-}$ anions [4]. As mentioned before, L-tryptophan and $\mathrm{CO}_{3}^{2-}$ ion probably generate the overlap of their corresponding bands, although this fact must be justified by theoretical models that we will leave for future reports. This absorption is surely a byproduct generated by the progenitor reagents in our growing conditions, probably due to fragmenting conditions of $\mathrm{Pb}\left(\mathrm{CH}_{3} \mathrm{COOH}\right)_{2}$ (see (2)). The band located at $\sim 1602 \mathrm{~cm}^{-1}$ could not be identified, but possibly it is a product generated by thiourea. The vibration of the amine group is associated with the bands corresponding to $\sim 3414 \mathrm{~cm}^{-1}$ (stretching in 

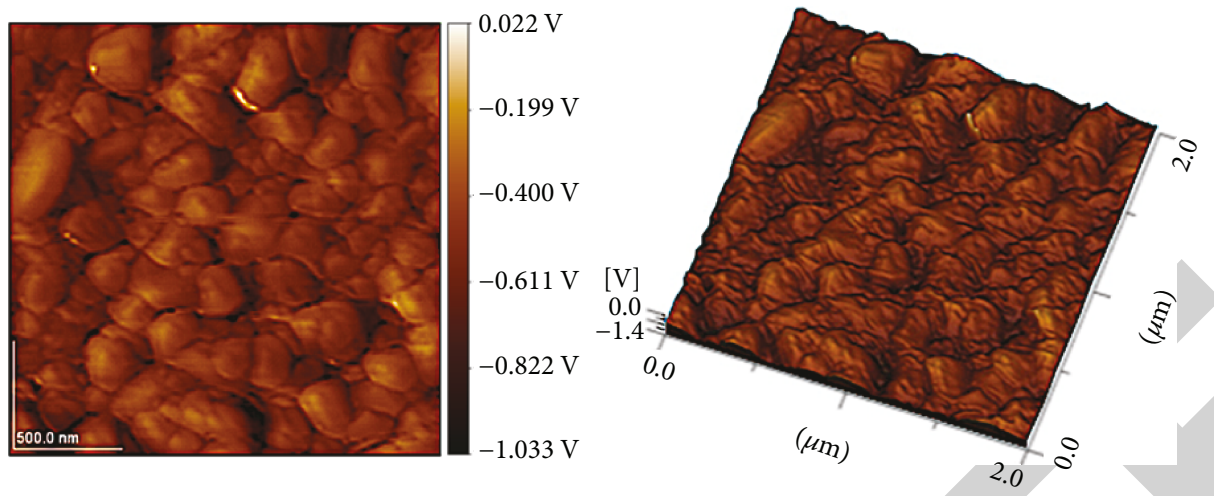

(a)
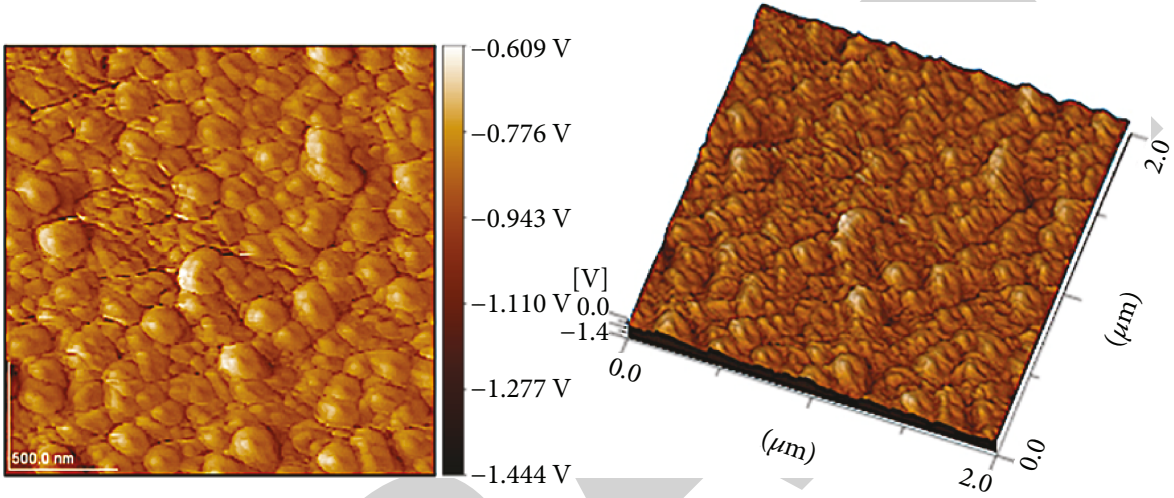

(b)
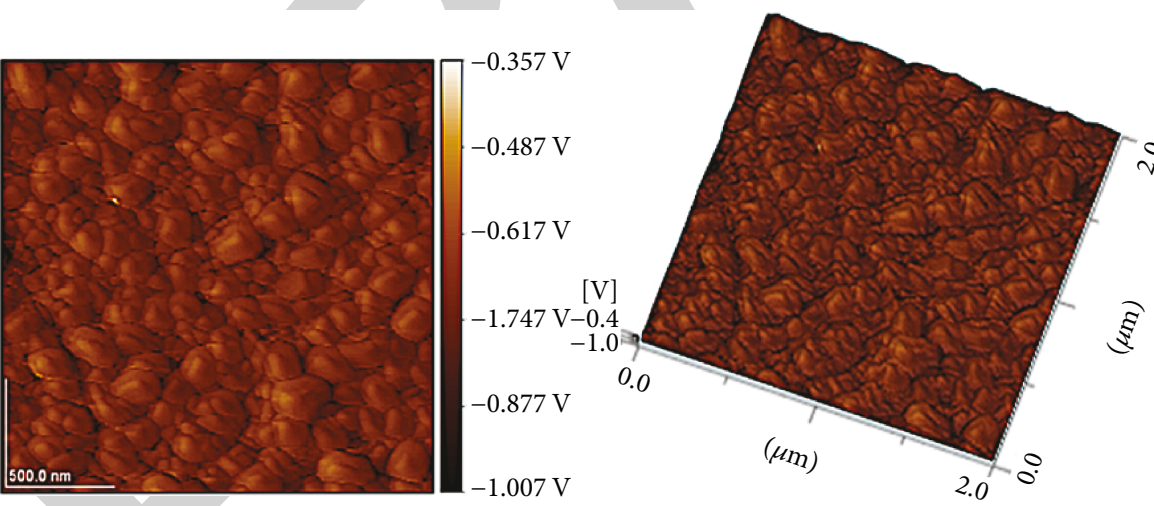

(c)
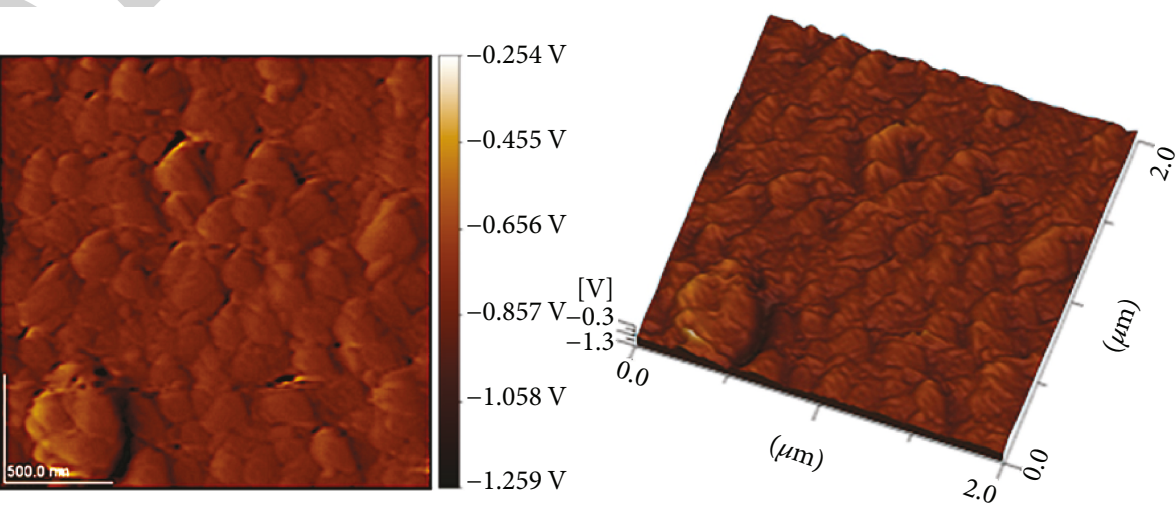

(d)

FIgURe 4: Continued. 

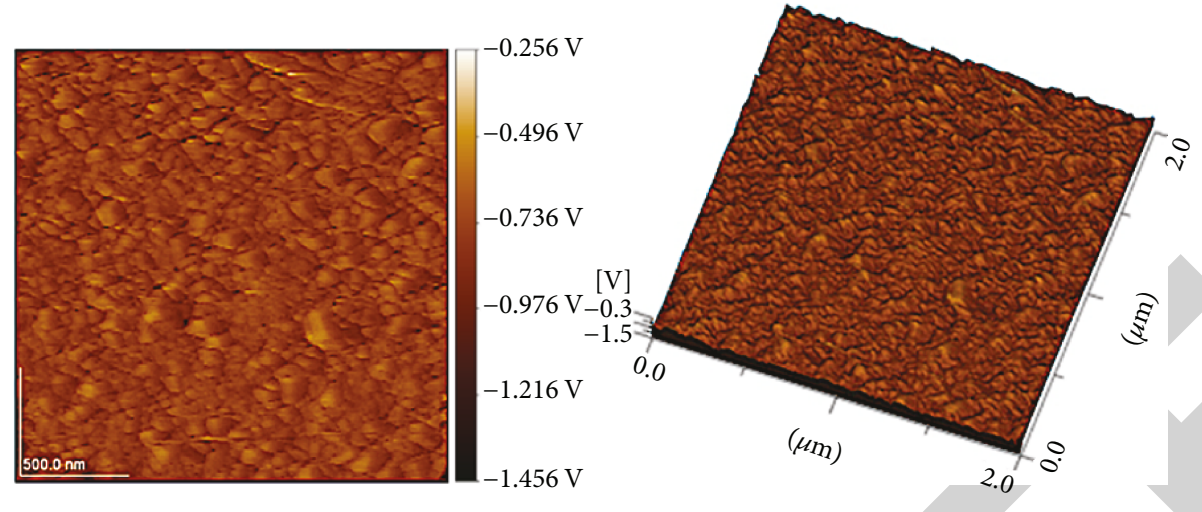

(e)

Figure 4: AFM II-D images for (a) PbS (b) PbS-T5 and (c) PbST10, (d) PbS-T20, and (e) PbS-T30 nanocrystals. Significant morphological changes are observed when compared to each other.

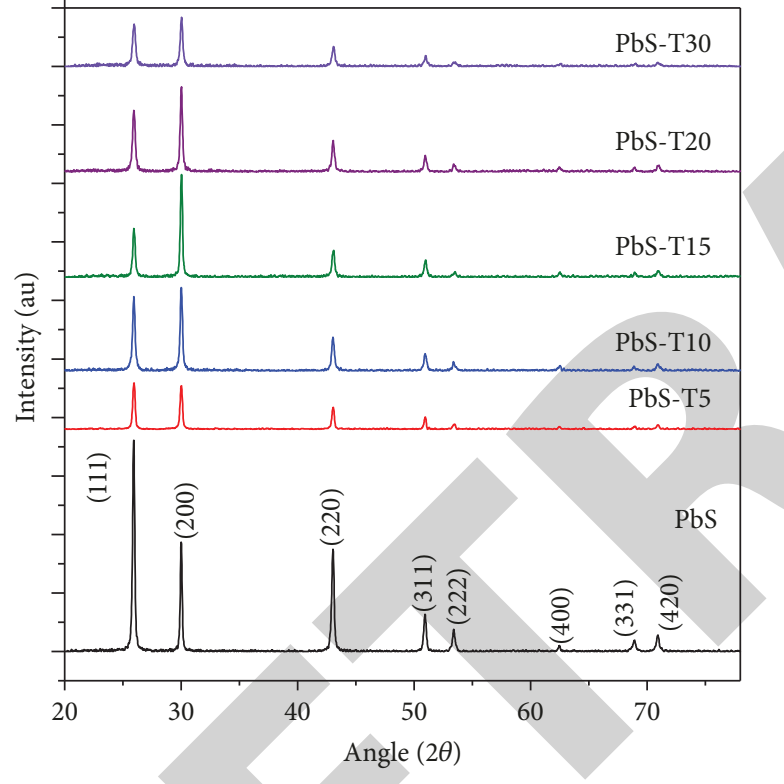

Figure 5: XRD diffractograms of PbS-PbS-T nanocrystals. A gradual change in orientation of the (111) and (200) crystalline plane can be seen in these diffractograms.

the amine group) $(>\mathrm{N}-\mathrm{H})$; that is, $-\mathrm{NH}_{2}$ groups are generated by the dissociation of the progenitor reagents $\left(\mathrm{NH}_{4} \mathrm{NO}_{3}\right)$, and the band at $\sim 1632 \mathrm{~cm}^{-1}$ with a small and broad band absorption located at $\sim 1920 \mathrm{~cm}^{-1}$ was assigned to one of the fundamental frequency absorptions of $\mathrm{O}=\mathrm{C}-$ $\mathrm{O}^{-}$anions [3]. An absorption band located at $\sim 2376 \mathrm{~cm}^{-1}$ can be seen and assigned to the $-\mathrm{C}=\mathrm{N}$ bond which could be generated possibly by the hydrolysis of thiourea under alkaline conditions. In the FTIR spectra corresponding to PbS$\mathrm{PbS}-\mathrm{T}$ films, a very intense broad band located at the $\sim 3450 \mathrm{~cm}^{-1}$ region can be associated to stretching of $\mathrm{OH}^{-}$ ions and defective sites of the physically adsorbed $\mathrm{H}_{2} \mathrm{O}$ molecules, associated to the alkaline medium. All PbS-PbS-T samples showed two vibration modes at $\sim 2855$ and $\sim 2928 \mathrm{~cm}^{-1}$ with less intensity corresponding to $\mathrm{CO}_{3}^{2-}$ anions [3]. These spectra clearly showed that many $\mathrm{OH}^{-}, \mathrm{O}=\mathrm{C}-\mathrm{O}^{-}$, and $\mathrm{CO}_{3}^{2-}$ anions remain on the surface of nanocrystals even after excessive washing as well as drying with hot air flow at $\sim 100^{\circ} \mathrm{C}$. Therefore, the bands associated with the Ltryptophan biomolecule cannot clearly be identified by the FTIR technique. This is stated by the fact that practically the bands observed in the $\mathrm{PbS}$ are also presented in the $\mathrm{PbS}$ - T nanocrystals. It is likely that these bands are more intense when they interfere with those of L-tryptophan. The bands observed in the thiourea solution when they are compared to those presented by PbS-PbS-T nanocrystals do not coincide and showed a shift towards a higher frequency. A plausible explanation is associated to the state of thiourea; in one case, the band corresponds to the solution and, in the films, they are in solid state, and therefore the shift in these bands is justifiable.

The surface images of the PbS-PbS-T thin films obtained by AFM are shown in Figure 4 for (a) PbS, (b) PbS-T5, (c) PbS-T10, (d) PbS-T20, and (e) PbS-T30 samples. These images showed significant changes when compared to each other. The PbS film showed that the grain growth is rather different from each other, indicating a regular growth rate of the crystal, and no holes are observed between grain boundaries. Likewise, there are grains overlapping each other on a uniform surface. In (b), (c) (d), and (e) images, the grain density was reduced indicating the presence of smaller nanocrystals. The surface roughness is very small, and this fact is modified almost gradually with $\mathrm{V}_{\text {[L-tryptophan] }}$. In (e), PbST30 images showed that the small spherical nanocrystals with an approximately $\sim 40-20 \mathrm{~nm}$ size were uniformly distributed over the smooth homogeneous background crystalline phase of $\mathrm{PbS}$, all layers presenting compact nanograins. In these images, well-defined borders can be observed and the effect of the incorporation of L-tryptophan in the films is clearly remarked.

$\mathrm{XRD}$ diffractograms for PbS-PbS-T nanocrystals are shown in Figure 5. Eight reflections can be observed demonstrating a randomly textured polycrystalline film, similar to those already reported [5]. These XRD diffractograms exhibited reflections located at $2 \theta \sim 26.08^{\circ}, 30.13^{\circ}, 43.08^{\circ}$, $51.91^{\circ}, 53.60^{\circ}, 62.51^{\circ}, 68.98^{\circ}$, and $71.15^{\circ}$ corresponding to 


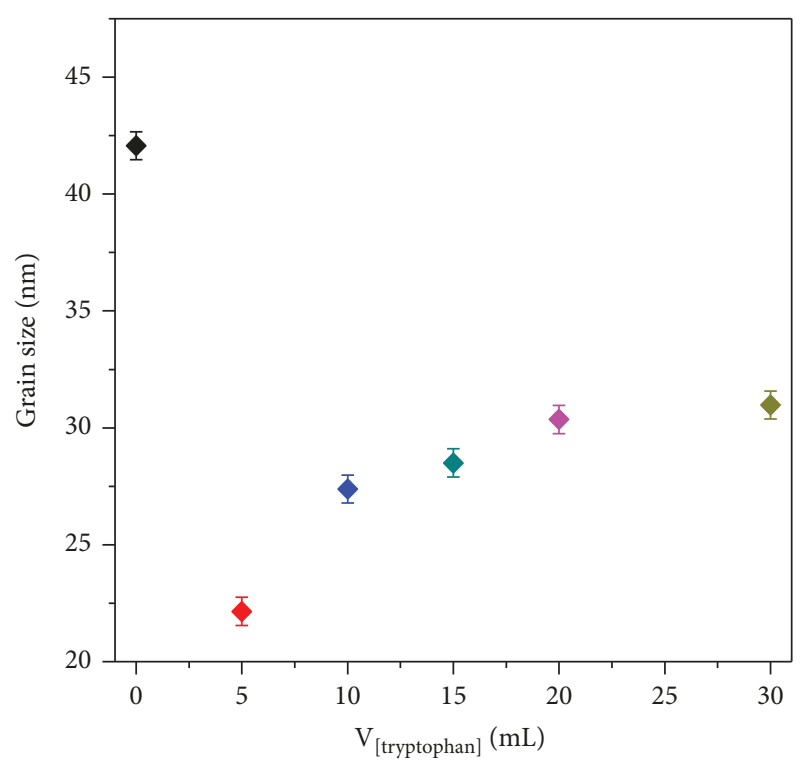

FIGURE 6: Average GS versus $V_{\text {[tryptophan] }}$ plot. An abrupt decrease with $\mathrm{V}_{\text {[L-tryptophan] }}$ and further decrease reaching saturation can be observed.

the crystalline planes (111), (200), (220), (311), (222), (400), (331), and (420), respectively, and all these reflections can be indexed to $\mathrm{PbS}$ nanocrystal diffractograms displaying the zinc blende (ZB) crystallinity phase (JCPDS 05-0592). These diffractograms show sharp reflection, indicating a polycrystalline nature. However, in these XRD diffractograms, a small shift of the crystalline planes to smaller values of $2 \theta$ in the PbS-T nanocrystal was observed. This shift in the diffraction lines can be attributed to lattice strain resulting from structural disorder generated by the doping effect. This shift in reflections is usually typical and is associated with the incorporation into the crystal lattice of the dopant ion $[3-5,10]$.

The shift induced by electrostatic repulsion can be associated to the electronic cloud in the organic molecule. On the other hand, the absence of other reflections corresponding to clusters and/or impurities induced a good crystallinity quality in the nanocrystals. XRD diffractograms for PbST15 and PbS-T20 films displayed a preferred orientation in the (200) reflection plane and has the highest intensity; this behaviour may be attributed to the L-tryptophan effect. This effect can be associated with molecular distribution, so the biomolecule shows a flat end that has a resonant effect correlated with $\pi \rightarrow \pi^{*}$ electron transition, as well as a chiral carbon on the other moiety of the molecule bearing the amino acid functional group [20]. The organic molecule possibly undergoes a flattening in the crystalline growth and simultaneously is surrounded by small nanocrystals (nanoparticles that have a dipole moment different from zero, when approaching the charge density of L-tryptophan, and this is modified by the electrostatic interaction producing distortion in the growth of nanocrystals). Thus, the electrostatic interactions induce an orientation change in the crystalline plane (111) presenting a preferential orientation of the (200) crystalline plane, as shown in Figure 5. Such a mechanism has been proposed for the crystalline growth with crystals having different morphologies, as discussed with the obtained AFM results. However, the incorporation in the crystalline lattice of molecules with the dimensions of L-tryptophan $(\sim 80 \mathrm{~nm})$ is difficult to explain considering its molecular structure. We propose that $\mathrm{PbS}$ nanocrystals are agglomerated around the L-tryptophan molecule generating nanocrystals; this phenomenon leads consequently to the growth of $\mathrm{PbS}$ nanocrystals. The average GS versus $\mathrm{V}_{\text {[L-tryptophan] }}$ plot for PbS-PbS-T films corresponding to the (111) crystalline plane is shown in Figure 6. A strong GS decrease for PbS$\mathrm{T} 5$ and then a gradual increase in PbS-T films can be observed, noticing that the GS reduces in the $5 \mathrm{~mL} \leq$ $\mathrm{V}_{\text {[L-tryptophan }]} \leq 30 \mathrm{~mL}$ range. For the undoped $\mathrm{PbS}$ film, a GS of $\sim 42 \mathrm{~nm}$ was obtained, and for PbS-T nanocrystals, the GS was obtained in the $\sim 22-30 \mathrm{~nm}$ range.

In several reports, doping is performed with positive and/or negative monovalent ions $\left(\mathrm{Li}^{+}\right)$and divalent $\left(\mathrm{Cu}^{2+}\right.$ or $\left.\mathrm{Se}^{2-}\right)$ and trivalent $\left(\mathrm{In}^{3+}\right)$ ions, as well as the simultaneous incorporation of $\mathrm{Cd}^{2+}, \mathrm{Bi}^{3+}$, and $\mathrm{Er}^{3+}$ ions into the crystal lattice of $\mathrm{PbS}$ [4], and, in all these reports, a GS decrease due to the effect of the dopant ion can be observed. A GS decrease is expected to lead to enhanced growth of stable nuclei in the initial growth stages, resulting consequently in smaller GS in the PbS-T nanocrystals. Worthy of mention is the unstable structure of PbS-T nanocrystals, which are incorporated surrounding the Ltryptophan molecule and then strain would arise, allowing L-tryptophan into the PbS nanocrystal to become a stable entity. In order to stabilize the crystal structure, the GS of $\mathrm{PbS}$ is reduced to release the strain. This behaviour reducing the GS of the nanocrystals is contrary to what is reported to be the usual when doping with ions that are introduced into the crystal lattice [3]. In the case of the L-tryptophan molecule, the situation is different since the molecule has a dimension of $\sim 80 \mathrm{~nm}$. In a report of CdO doped with an organic molecule of greater molecular weight and size (coumarin) [13], the authors associate the incorporation of a coumarin molecule into the crystalline network by replacing oxygen, but the distance of both lattice parameter $(a)$ and the interplanar distance (ID) is one hundred times smaller than the size of the host molecule (see Figures 7(a) and 7(b)). We consider then, according to the molecular dimensions, that such explanation is questionable.

The model that we propose has a very different concept: we associate the crystalline growth with the electrostatic interaction of the $\mathrm{PbS}$ nucleus to the electronic cloud $(\pi$ electrons) of the tryptophan biomolecule. In Figure 7, (a) ID versus $\mathrm{V}_{\text {[L-tryptophan] }}$ and (b) $a$ versus $\mathrm{V}_{\text {[L-tryptophan] }}$ of $\mathrm{PbS}-\mathrm{PbS}-\mathrm{T}$ nanocrystals are shown. In these two plots, a quasilinear behaviour can be seen, except for a small shift in the PbS-T20 layer. Both cobehaviours are similar and there is a gradual decrease in ID and $a$, respectively; thus, it is clear that L-tryptophan is not incorporated into the crystalline network and the effect produces a gradual widening in ID and $a$, respectively. That is, the formation of clusters where L-tryptophan surrounds and compresses the crystal lattice may be possible because the size of 


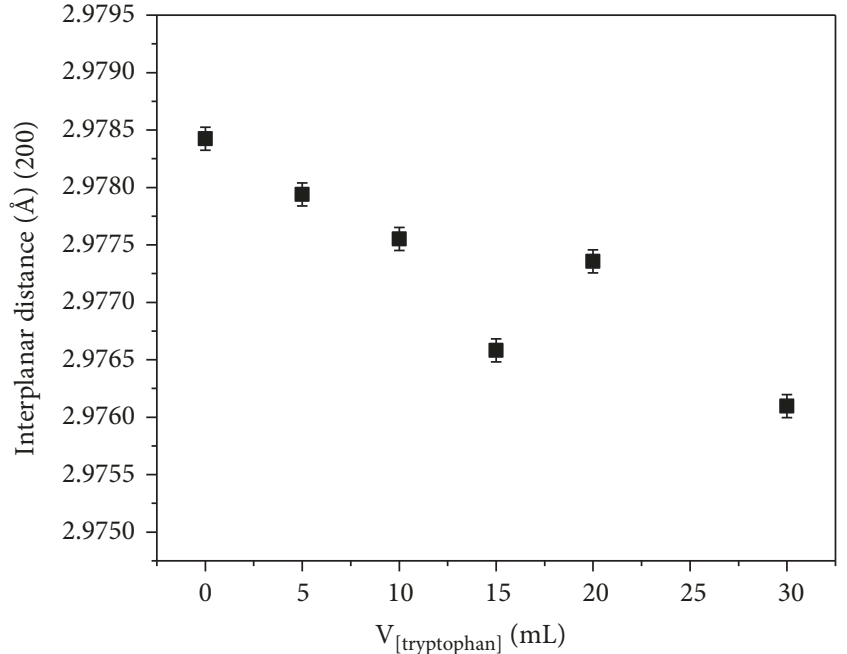

(a)

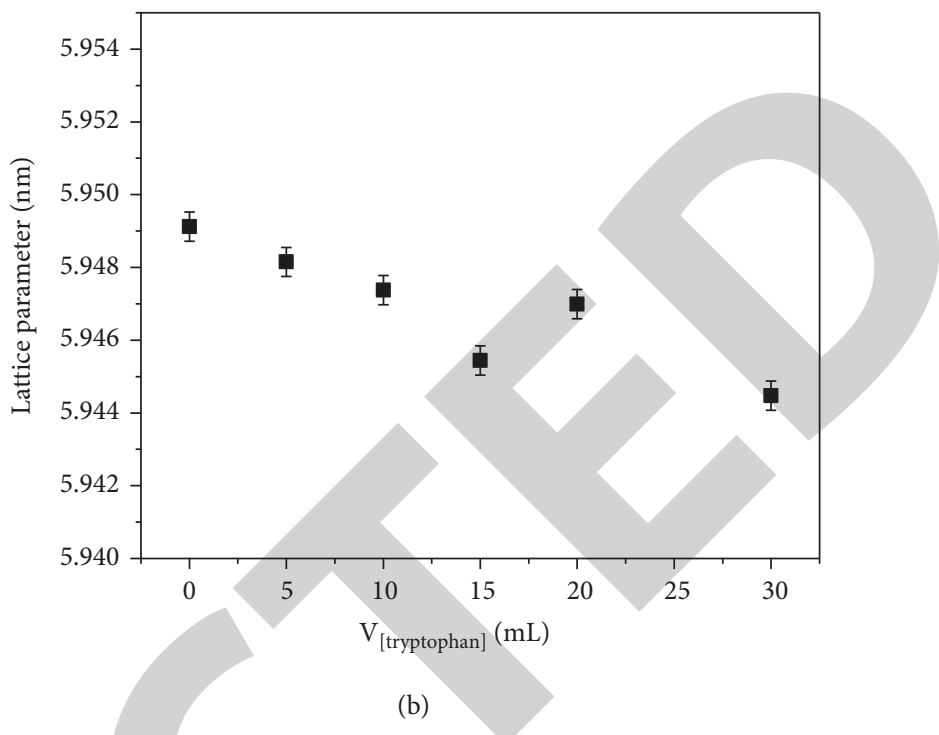

Figure 7: (a) Interplanar distance (ID) and (b) lattice parameter of PbS-PbS-T nanocrystals. A gradual widening of these crystalline parameters can be observed.

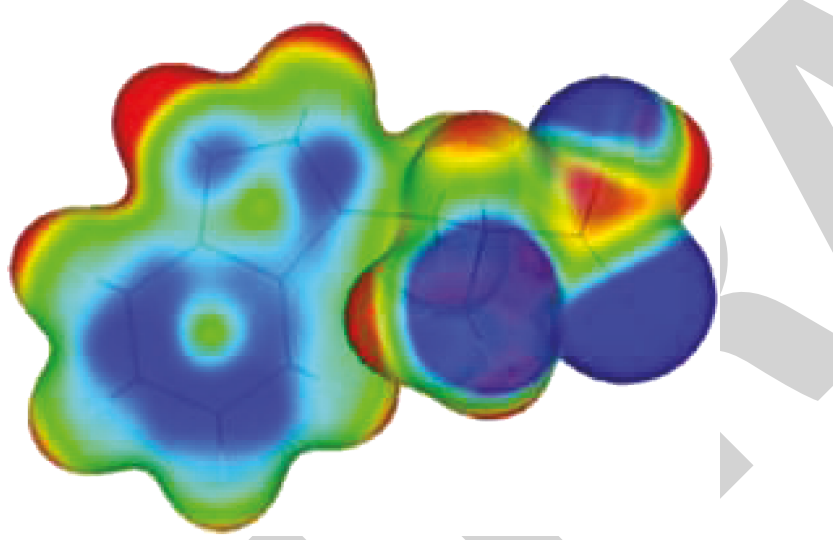

Figure 8: Molecular electrostatic potential (MEP) mapped onto an isosurface of the total electron density for the L-tryptophan molecule.

L-tryptophan is larger than the $\mathrm{PbS}$ nanocrystal, which in turn implies a reduction in grain size as Ltryptophan's concentration grows.

By using different $\mathrm{V}_{\text {[L-tryptophan], we can control the level }}$ of doping and GS, respectively. Below, we present an empirical and plausible answer to the origin of the GS decrease in the PbS nanocrystal. In order to understand the effect of Ltryptophan on crystal growth, we used a theoretical diagram of the electron density distribution associated with the $\pi$ electrons. We deemed the electronic charge distribution density of L-tryptophan using the molecular electrostatic potential (MEP) based on the static charge distributions of the nuclei and electrons within it, which can be analyzed to predict the reactivity of the molecule [21].

This potential has also proven to be a particularly useful indicator of the sites or regions of a molecule to which an approaching electrophile or nucleophile would be attracted. It has been applied successfully to study interactions between reactants and for recognition in biological systems (e.g., in enzyme-substrate systems and on molecular surface) [22]. In Figure 8, the MEP mapped onto an isosurface of the total electron density for the L-tryptophan is shown, where blue regions are observed on the moiety corresponding to the conjugated rings, as well as to the amino acid functional group indicating higher negative charge.

With the PbS nanocrystal ( $42 \mathrm{~nm})$, the MEP behaviour of the aryl group is slightly positive (red color); this charge density significantly alters the GS of crystals in the $\mathrm{PbS}$, although the electric interaction in the solution is difficult to explain. However, a first approximation using this model allows explaining the origin in an approximate and semiempirical way of the gradual GS decrease.

In order to calculate strain $(\varepsilon)$, the well-known Williamson-Hall equation was considered [4], where $\varepsilon$ of GS is related to the measured full width at half maximum ( $\beta$ ) of the diffraction peaks by following the $\beta \cos \theta=\lambda$ / $\mathrm{GS}+\varepsilon \lambda \operatorname{sen} \theta$ equation, where $\lambda$ is the wavelength of the $\mathrm{X}$-ray source and $\theta$ is Bragg's angle, respectively. The $\beta$ $\cos \theta$ versus $\operatorname{sen} \theta$ plot of PbS-PbS-T nanocrystals is displayed in Figure 9. In these plots, the behaviour is not linear. In general, the slope of the plot gave the amount of residual $\varepsilon$. Therefore, $\varepsilon$ can produce defects and structural distortions in the nanocrystal, for example, shrinking in the crystalline lattice constants. The thickness, GS, roughness, stoichiometric ratios, grain boundaries, and stacking faults are parameters associated with $\varepsilon$ in semiconductor films [23]. The changes presented in $\varepsilon$ for this material showed significant differences between them as mentioned before. The L-tryptophan molecule in crystal growth probably modifies its molecular configuration, although investigating these changes requires a deeper study that is outside our objective in the present report.

The behaviour of $\varepsilon$ is associated with the incorporation of L-tryptophan. Incorporation is difficult to explain empirically although the anisotropic change is evident in these plots. Consequently, structural changes as well as the 


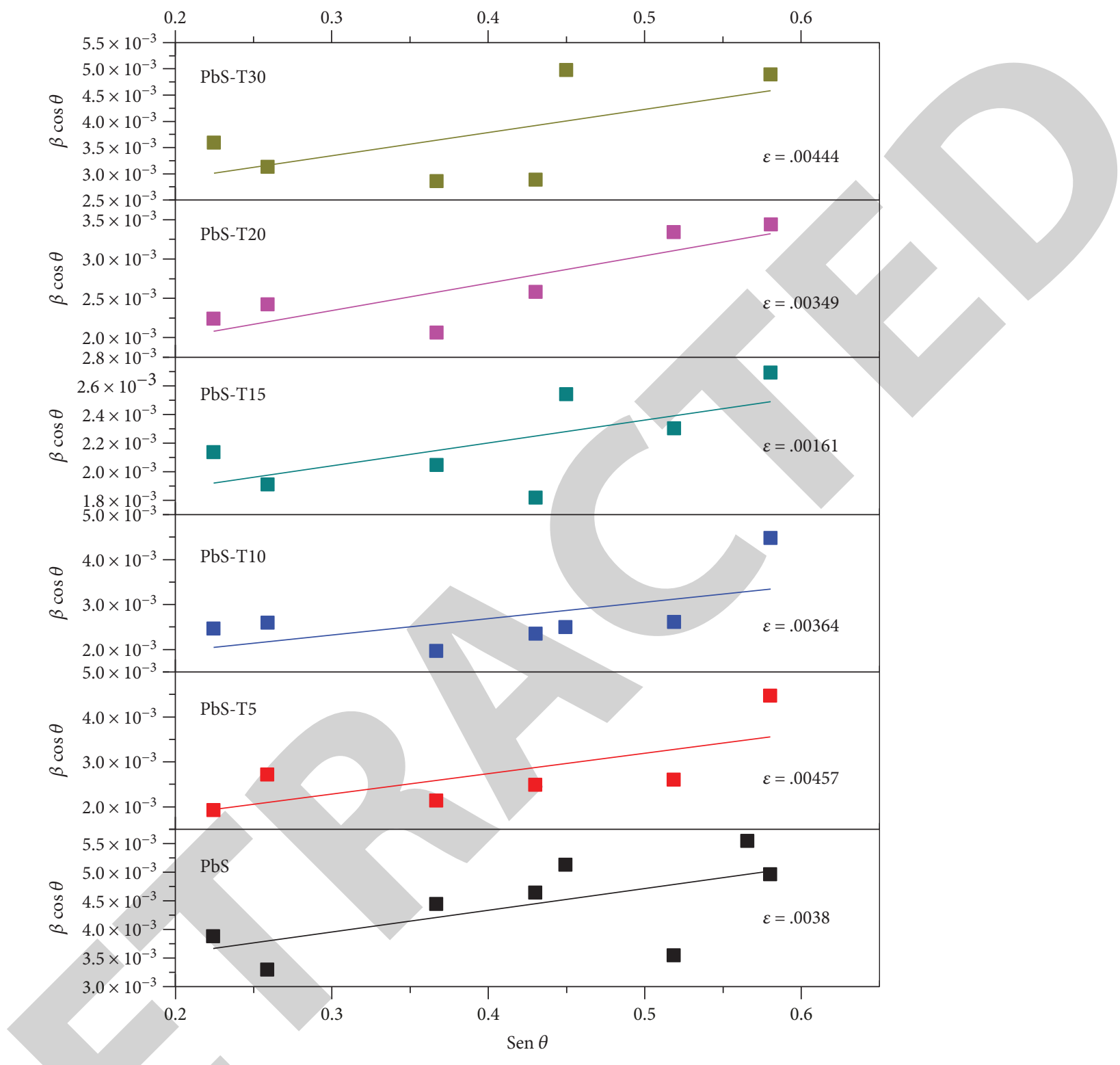

FIGURE 9: $\beta \cos \theta$ versus $\operatorname{sen} \theta$ plot of PbS-PbS-T nanocrystals. In this plot, changes in the crystal structure associated with the incorporation of L-tryptophan, showing stress in all the nanocrystals, can be observed.

positions of the corresponding ions in the crystal lattice of $\mathrm{PbS}$ inside the crystallites are associated to the grain boundary, which dissipates and leads to a reduction in the concentration of lattice imperfections. A strain versus $\mathrm{V}_{\text {[L-tryptophan] }}$ plot of PbS-PbS-T nanocrystals is shown in Figure 10. Like the GS versus $\mathrm{V}_{\text {[L-tryptophan] }}$ plot, a drastic change by incorporation of $\mathrm{V}_{\text {[L-tryptophan] }}$ in the $\mathrm{PbS}$ matrix can be observed. This is an interesting point because anisotropy could be modified systematically, although this behaviour has demonstrated that a hybrid film can show an anisotropic response after the confinement of semiconductor macromolecules in the oriented mesochannels [24]. Similar results were observed when dye molecules or metal nanowires were introduced into the oriented mesochannels [25].

The experimental results showed that there is a residual $\varepsilon$ and the PbS-T films displayed a behaviour associated with the interaction of the surfaces that present a different anisotropy. From these experimental results, the behaviour in the dispersed numerical values of $\varepsilon$ is associated with the disordered incorporation of the L-tryptophan molecule that induces drastic changes in PbS anisotropy. Generally, the change in strain is associated with structural (crystalline) changes; in this report, an abrupt change (decrease) is firstly observed and then it remains constant and finally increases. Probably the structural distortion in the crystalline growth of L-tryptophan causes different orientations in the crystalline structure of the $\mathrm{PbS}$, and this is associated with the electrostatic repulsions. Dislocation density $(\delta)$ is defined as the length of dislocation lines per unit volume of the crystal, and higher $\delta$ values indicate lower crystallinity levels for the crystalline material [26]. The following $\delta=1 / G S$ equation allows evaluating 


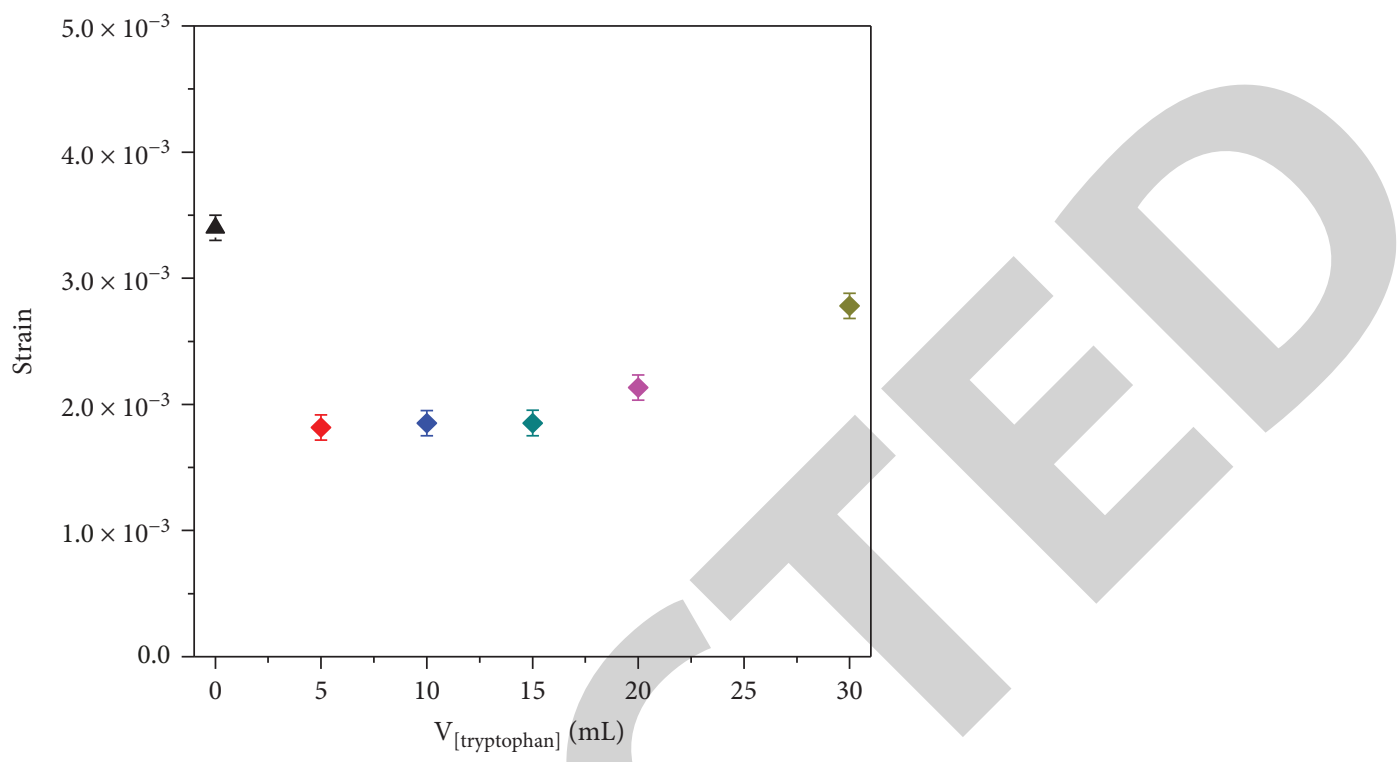

FIGURE 10: Strain versus $\mathrm{V}_{\text {[tryptophan] }}$ plot of PbS-PbS-T nanocrystals. A drastic change is observed by the incorporation of $\mathrm{V}_{\text {[tryptophan] }}$ into the $\mathrm{PbS}$ matrix.

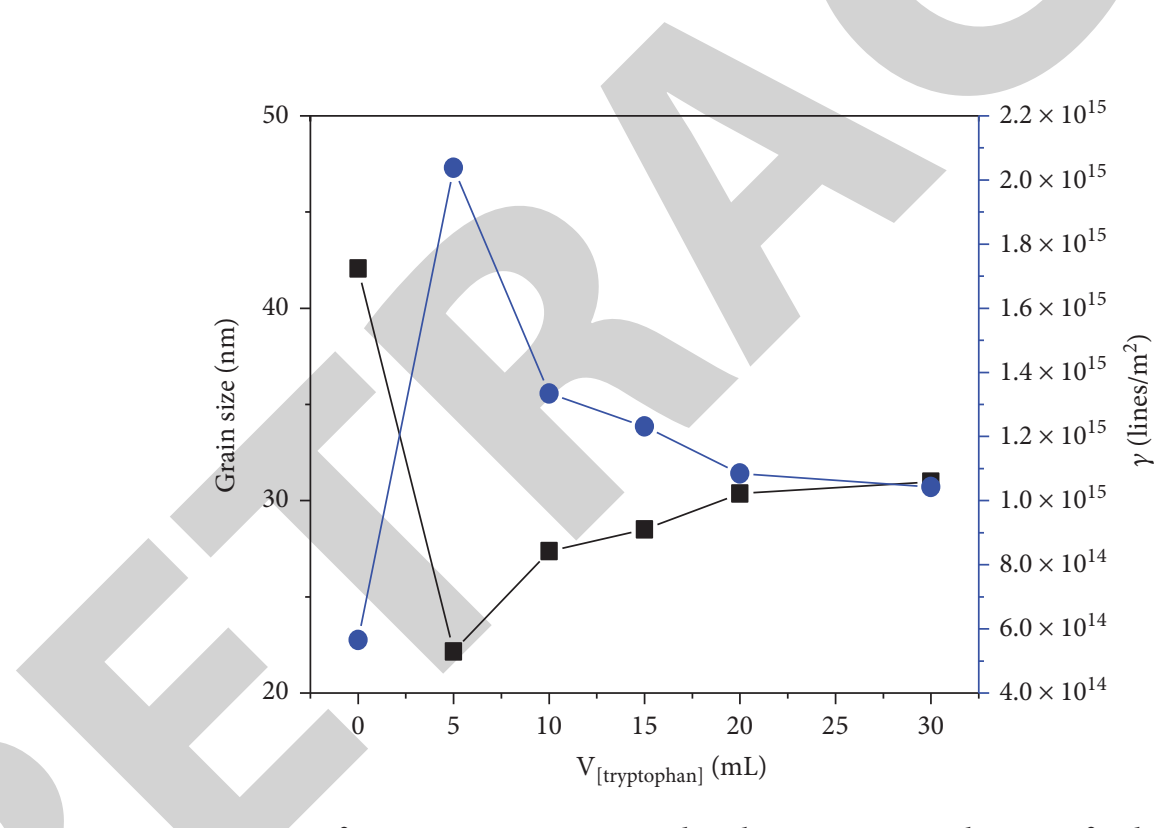

FIGURE 11: $\delta$-GS versus $\mathrm{V}_{\text {[L-tryptophan] }}$ plot, showing a strong change in $\delta$ value.

the experimental value of $\delta$. Figure 11 shows the $\delta$-GS versus $\mathrm{V}_{\text {[L-tryptophan] }}$ plot.

This plot shows a strong change in $\delta$ value and then remains with little significant changes, almost reaching a saturation level. Therefore, the number of defects increases gradually with the $\mathrm{V}_{\text {[L-tryptophan] }}$ increase as expected. The GS decrease presents an increase in the grain boundaries and in the number of defects in the crystalline structure.

The optical properties were also examined to find the correspondence with the structural and morphological properties previously investigated. The reflectance spectra of PbS-PbS-T films were obtained and analyzed over the 200-1500 $\mathrm{nm}$ wavelength range at RT. The reflectance versus wavelength $(\mathrm{nm})$ spectra of $\mathrm{PbS}-\mathrm{PbS}-\mathrm{T}$ nanocrystals are shown in Figure 12. A gradual decrease in reflectance is observed in the $\sim 350-450 \mathrm{~nm}$ range, and a high increase in $\sim 450-850 \mathrm{~nm}$ in PbS-T corresponding to the visible region can be observed. This low absorbance may be due to a decrease in grain boundary scattering due to a GS enhancement considering the thickness as another parameter in the optical analysis for the materials. One plausible explanation for this optical behaviour in thin films is considering the surface roughness as having a great effect on the optical measurements, mainly at wavelengths (emeries) close to electronic transitions. In these spectra, the electronic transitions are observed because the GS dimensions are closer to the excitonic radius of PbS. Typically, Ltryptophan has a wavelength of minimum reflectance at $\sim 280 \mathrm{~nm}(\sim 4.4 \mathrm{eV})$ and an emission peak that is 


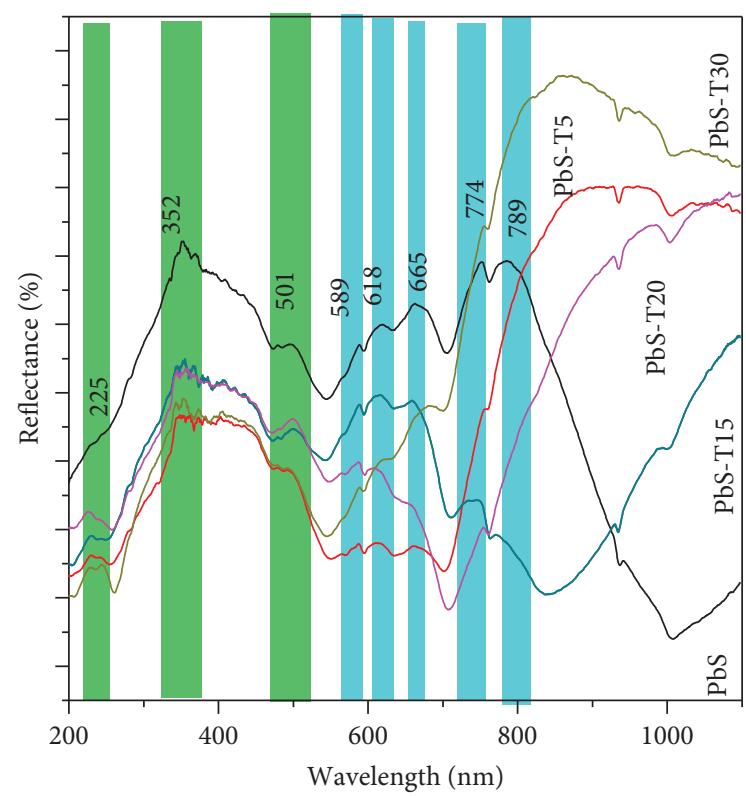

FIGURE 12: Reflectance versus wavelength spectra from the 200 to $1500 \mathrm{~nm}$ range of $\mathrm{PbS}-\mathrm{PbS}-\mathrm{T}$ films. The spectra of the PbS$\mathrm{T}$ films show an absorbance decrease when compared to the PbS nanocrystals.

solvatochromic, ranging at ca. $\sim 300-350 \mathrm{~nm}(\sim 4.1-3.5 \mathrm{eV})$, depending on the polarity of the local environment [27].

In the experimental results shown, it is not possible to clearly differentiate the bands associated with L-tryptophan; however, changes in the optical response of the PbS nanocrystal were observed. From these experimental observations, it is common to associate this effect of L-tryptophan with a catalyst in the crystalline growth, which inhibits the increase (volume and length) of crystal. The absorbance spectra of PbS-PbS-T nanocrystals exhibited low absorption bands (bands shown in green and blue color) located at $\sim 225 \mathrm{~nm}$ $(\sim 5.5 \mathrm{eV}), \sim 352 \mathrm{~nm}(\sim 3.6 \mathrm{eV}), 501 \mathrm{~nm}(2.4 \mathrm{eV}), \sim 589 \mathrm{~nm}$ $(\sim 2.1 \mathrm{eV}), \sim 618 \mathrm{~nm}(\sim 2.0 \mathrm{eV}), \sim 665 \mathrm{~nm}(\sim 1.8 \mathrm{eV}), \sim 774 \mathrm{~nm}$ $(\sim 1.6 \mathrm{eV})$, and $\sim 789 \mathrm{~nm}(\sim 1.5 \mathrm{eV})$ due to a strong confinement effect $[3-6,10,28]$. It can be seen that $\mathrm{PbS}$ exhibited a strong and sharp absorption band located at $\sim 352 \mathrm{~nm}$ $(\sim 3.6 \mathrm{eV})$, which is fairly blue-shifted from the absorption edge of bulk $\mathrm{PbS}(\sim 3020 \mathrm{~nm})$ already reported [29], because the UV absorption decreases dramatically below the wavelength corresponding to the band edge. It has been reported that this kind of band edge luminescence arises from the recombination of excitons shallowly trapped in electronhole pairs [30]. The alkoxy radical is a well-known decomposition product of many carboxylic acids and displays a signature absorption profile ranging from $\sim 500$ to $650 \mathrm{~nm}(\sim-1.9-2.4 \mathrm{eV})$; see (1) and (2) [31]. Such effect is associated with the L-tryptophan molecule as well as the $\mathrm{CO}_{3}^{2-}$ ions that may be present on the surface, as well as the volume of these nanocrystals. The transition band located at $\sim 225 \mathrm{~nm}(\sim 5.51 \mathrm{eV})$ cannot be identified, but it can be associated with the incorporation of L-tryptophan. In our opinion, this optical and structural behaviour requires an analysis through the application of some

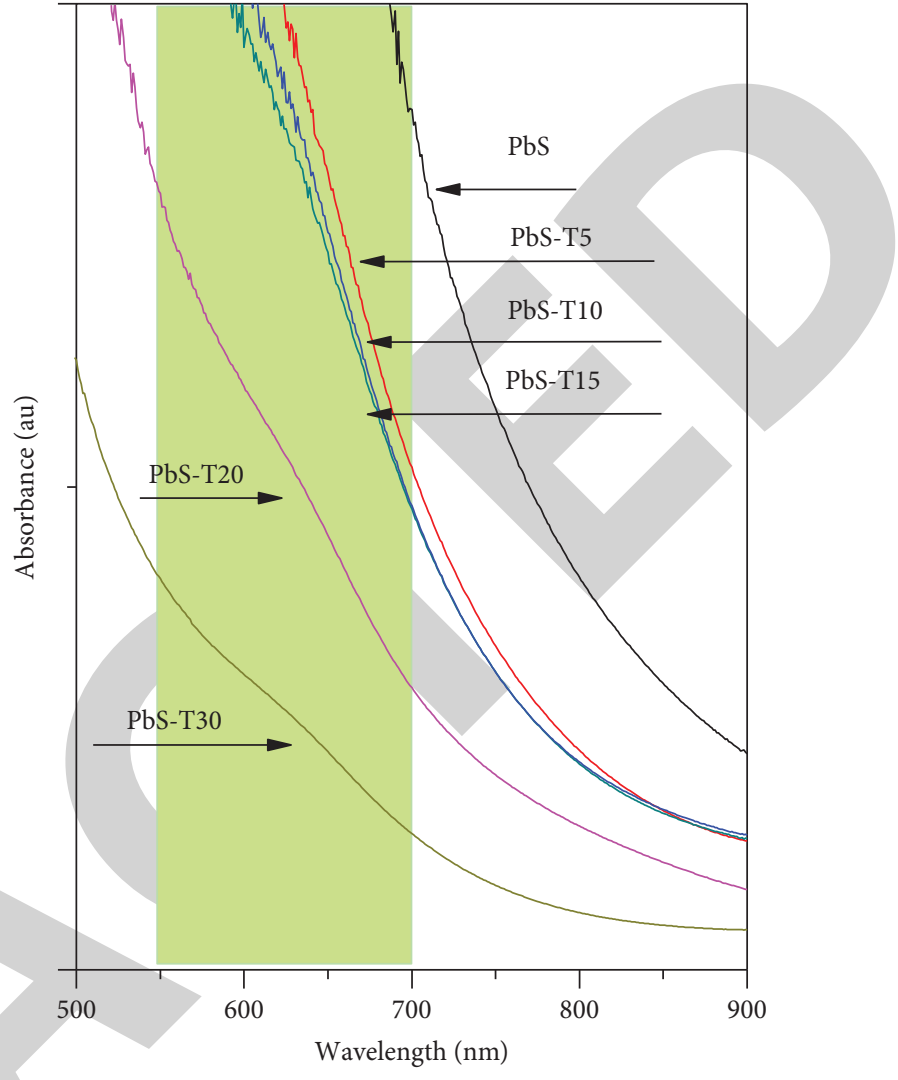

FIgURE 13: Absorbance spectra at the $\sim 500-900 \mathrm{~nm}$ range of PbS$\mathrm{PbS}-\mathrm{T}$ films, with $\mathrm{PbS}-\mathrm{T}$ films showing a decrease in absorbance when compared to the PbS film.

theoretical models as mentioned before and differentiating interactions of L-tryptophan with $\mathrm{PbS}$ nanocrystals; this report only presents the experimental results and a semiempirical analysis. The excitonic band located at $\sim 665 \mathrm{~nm}(\sim 1.8 \mathrm{eV})$ is generally assigned to the ${ }^{1} \mathrm{~S}_{\mathrm{h}} \rightarrow{ }^{1} \mathrm{~S}_{\mathrm{e}}$ transition [32]. Though there has been debate over assignment of the excitonic transition at $\sim 1.4 \mathrm{eV}(\sim 885 \mathrm{~nm})$, it is generally considered as a ${ }^{1} \mathrm{P}_{\mathrm{h}} \rightarrow{ }^{1} \mathrm{P}_{\mathrm{e}}$ transition band and we do not issue any comments on that statement. The band located at $\sim 789 \mathrm{~nm}(\sim 1.5 \mathrm{eV})$ is probably generated to the electronic transitions associated with the excitonic band located at $\sim 774 \mathrm{~nm}(\sim 1.6 \mathrm{eV})$. On the other hand, the bands at $\sim 665 \mathrm{~nm}(\sim 1.8 \mathrm{eV})$ and $\sim 589 \mathrm{~nm}(\sim 2.1 \mathrm{eV})$ are due to higher energy transitions from ${ }^{1} D_{\mathrm{h}} \rightarrow{ }^{1} D_{\mathrm{e}}$ and ${ }^{2} S_{\mathrm{h}} \rightarrow{ }^{2} S_{\mathrm{e}}$, respectively [33,34]. Regarding the excitonic absorption band reported at $\sim 618 \mathrm{~nm}(\sim 2.0 \mathrm{eV})$, it is well-known that this peak is strongly related to surface change separation and polarization effects and is thus sensitive to charges on the surface molecules [35]. The shift seen in some transitions is associated with the polarization of L-tryptophan and impurities incorporated during crystalline growth. We associate this molecular effect to the presence of the L-tryptophan molecule that is surrounded by $\mathrm{PbS}$ nanoparticles according to our semiempirical model proposed. There have been reports about a very large Stokes shift in $\mathrm{PbS}$ quantum dots, which was attributed to the presence of localized surface states or trap 
states [6]. The absorbance versus wavelength spectra ranging from $\sim 564$ to $729 \mathrm{~nm}(\sim 1.7-2.2 \mathrm{eV})$ for PbS-PbS-T films are shown in Figure 13.

A typical absorbance spectrum of PbS-PbS-T samples in the $\sim 500-900 \mathrm{~nm}$ (visible region) wavelength range is presented. This interval was chosen to examine the typical electronic transition bands associated with the quantum confinement effect. The PbS-T20 and PbS-T30 nanocrystals showed a wide and intense band located at $\sim 550-680 \mathrm{~nm}$ $(\sim 2.2-1.8 \mathrm{eV})$. These bands are shown with a green row for a rapid appreciation and generally can be identified with electronic transition in nanocrystalline $\mathrm{PbS}$ [36] induced by the widening of the $\mathrm{E}_{\mathrm{g}}$ (energy levels by GS decrease). In other words, the GS is approximately the size of the exciton radius and this electronic transition can be appreciated although it is not a very intense band. This transition band has been reported in doped-cobaltocene $\mathrm{PbS}$ and $\mathrm{PbSe}$ and is associated with the charge transference of such molecule, because of mirror symmetric valence and conduction bands in $\mathrm{PbS}$, with these transitions having the same energy and strength [11]. Such behaviour can be associated considering the following plausible explanation. The redox potential for oneelectron oxidation of L-tryptophan, as well as for a few simple indoles and phenols, has been determined, with the value for L-tryptophan $(\varepsilon=1.0 \mathrm{~V}$ versus NHE at $\mathrm{pH} 7.0)$ [37]. Due to the uncertainty about the redox potential for the biomolecule which lies above the conduction band edge of bulk $\mathrm{PbS}(0.1 \mathrm{~V}$ versus SHE) [38], we should expect that the electron transfer from L-tryptophan to the $\mathrm{PbS}$ nanocrystal is energetically favourable and significant changes in these electronic transitions are energetically probable.

Once the electrons are transferred to the ${ }^{1} \mathrm{~S}_{\mathrm{e}}$ conduction band state, the amplitude of the ${ }^{1} \mathrm{~S}_{\mathrm{e}}$ peak is expected to be reduced due to Pauli blocking. To visualize in detail, the transitions are presented by the $\mathrm{PbS}$ nanocrystal in such region. A deconvolution was carried out in the Gaussians corresponding to the PbS-PbS-T layers. The deconvolution of absorbance spectra in the range of $\sim 1.85-2.07 \mathrm{eV}(\sim 564-729 \mathrm{~nm})$ for (a) PbS-PbS-T, (b) PbS-T5, (c) PbS-T-T10, (d) PbS-T20, and (e) PbS-30 nanocrystals is shown in Figure 14. All these bands showed three transitions with a small shift. Gaussian line fitting of a PbS-T film shows, as the origin of the absorbance anisotropy, a weak contribution due to the Ltryptophan molecule featuring the dominant Gaussian curve. It is observed here that in the PbS-20 sample, the contribution is somewhat intense; this behaviour can be associated with the results of ID and $a$. This film presents a sharp jump with respect to all the samples according to Figures $7(\mathrm{a})$ and $7(\mathrm{~b})$, respectively. The components are most probably associated with the lifting of the degeneracy due to coupling of equivalent L-valleys and splitting of the lowest electronic transition in $\mathrm{PbS}-\mathrm{T}$ nanocrystals. In regard to the excitonic absorption peak reported at $\sim 580 \mathrm{~nm}(\sim 2.1 \mathrm{eV})$, it is well-known that this peak is strongly related to surface change separation and polarization effects and thus is sensitive to charges on the surface of molecules [39], and the excitonic peaks located at $\sim 1.8 \mathrm{eV}$ and $\sim 2.1 \mathrm{eV}$ are due to higher energy ${ }^{1} \mathrm{~S}_{\mathrm{e}} \rightarrow{ }^{1} \mathrm{~S}_{\mathrm{h}}$ and ${ }^{1} \mathrm{P}_{\mathrm{e}} \rightarrow{ }^{1} \mathrm{P}_{\mathrm{h}}$ transitions, respectively [10]. The diagram for ${ }^{1} \mathrm{D}_{\mathrm{h}} \rightarrow{ }^{1} \mathrm{D}_{\mathrm{e}},{ }^{1} \mathrm{~S}_{\mathrm{h}} \rightarrow{ }^{1} \mathrm{P}_{\mathrm{e}},{ }^{1} \mathrm{P}_{\mathrm{h}} \rightarrow{ }^{1} \mathrm{~S}_{\mathrm{e}}$, and ${ }^{1} \mathrm{~S}_{\mathrm{h}} \rightarrow{ }^{1} \mathrm{~S}_{\mathrm{e}}$ transitions is shown in Figure 15. The excitonic peaks at $1.85 \mathrm{eV}\left({ }^{2} \mathrm{~S}_{\mathrm{h}} \rightarrow{ }^{2} \mathrm{~S}_{\mathrm{h}}\right)$ and $\left.2.15 \mathrm{eV} /{ }^{2} \mathrm{P}_{\mathrm{h}} \rightarrow{ }^{2} \mathrm{P}_{\mathrm{e}}\right)$, respectively, are due to higher energy transitions [40].

Different reports have examined computational models about the GS decrease generating energetic levels within the band gap energy $\left(E_{g}\right)$ and the materials presenting the effect of quantum confinement. Quantized energy levels of PbSPbS-T nanocrystals are shown in Figure 15.

The experimental optical data were analysed from the tau relation $\alpha \mathrm{h} v=A\left(\mathrm{~h} v-\mathrm{E}_{\mathrm{g}}\right)^{n / 2}$ where $\mathrm{h} v$ is the photon energy, $\mathrm{E}_{\mathrm{g}}$ is the band gap energy, and $A$ and $n$ are constants. $A$ is related to the effective masses associated with the valence and conduction bands [41]. However, the PbS sample has a direct $\mathrm{E}_{\mathrm{g}}$; therefore, the constant $n$ is equal to 1 . The variation of $(\alpha \mathrm{h} v)^{2}$ with $\mathrm{h} v$ for PbS-PbS-T is a straight line which confirms the direct transition. $(\alpha \mathrm{h} v)^{2}$ versus $\mathrm{h} v$ plot of the PbS$\mathrm{PbS}-\mathrm{T}$ nanocrystal is shown in Figure 16. $\mathrm{E}_{\mathrm{g}}$ was determined by extrapolating the straight-line portion to the energy axis for cero absorption coefficient $\alpha$.

The $\mathrm{E}_{\mathrm{g}}$ versus $\mathrm{V}_{\text {[L-tryptophan] }}$ plot of PbS-PbS-T thin films is shown in Figure 17, and it was found to be $\sim 1.4-2.1 \mathrm{eV}$. Experimental numerical values of $\mathrm{E}_{\mathrm{g}}$ of $\mathrm{PbS}$-PbS- $\mathrm{T}$ thin films are shown in Table 1. This shift may be due to the quantum confinement effect in nanocrystalline films [42]. The $\mathrm{E}_{\mathrm{g}}$ value clearly increases gradually with $\mathrm{V}_{[\mathrm{L} \text {-tryptophan], }}$, particularly in the PbS-T30 sample. The confinement effect appears as a shift in the edge of the absorption spectra and the absorption to lower wavelengths due to the GS decrease. There is a decrease in number of defects as well as roughness on the surface and $\mathrm{H}_{2} \mathrm{O}$ molecules and $\mathrm{CO}_{3}^{2-}$ ion that adhere strongly both on the crystal surface and on the volume itself. The $\mathrm{E}_{\mathrm{g}}$ for PbS samples with $\sim 1.4 \mathrm{eV}(\mathrm{PbS})$ and two transitions of $\sim 1.5 \mathrm{eV}$ and $\sim 2.1 \mathrm{eV}$ are observed in the PbS-T30 film. The $\mathrm{E}_{\mathrm{g}}$ increase when increasing $\mathrm{V}_{\text {[L-tryptophan] }}$ in the films is reflected by the presence of an excitonic structure material. Excitonic structures are readily observed in large E semiconductors with binding energy [43]. The shift observed in the position of the excitonic peak towards higher energies in the PbS nanocrystal has been explained due to a doping effect [44]. It is clear that $\mathrm{E}_{\mathrm{g}}$ increases when $\mathrm{V}_{\text {[L-trypto- }}$ phan] increases, observing a strong confinement in doped PbS-T films. The optical and structural properties showed worth-noting changes in addition to the effect of strong quantum confinement observed in this semiconductor when doped systematically with the L-tryptophan biomolecule.

\section{Conclusions}

The effect of the dopant promotes the GS decrease, and this fact induces an $\mathrm{E}_{\mathrm{g}}$ shift towards greater energy. The structural properties are directly associated with the optical properties, and consequently, the existence of the effect of quantum confinement presented in this material is generated by the Ltryptophan biomolecule with effect on the crystalline growth during the nanocrystal formation. In summary, we have found an efficient process to introduce the L-tryptophan 


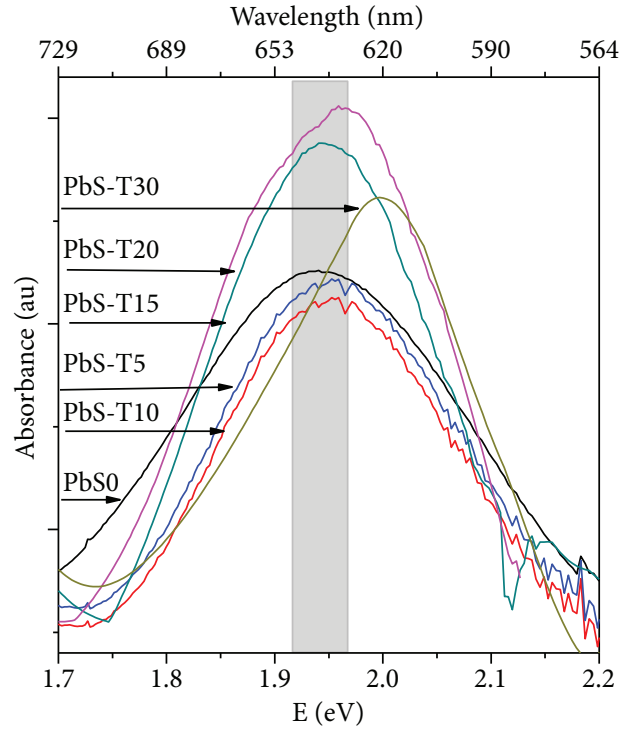

(a)

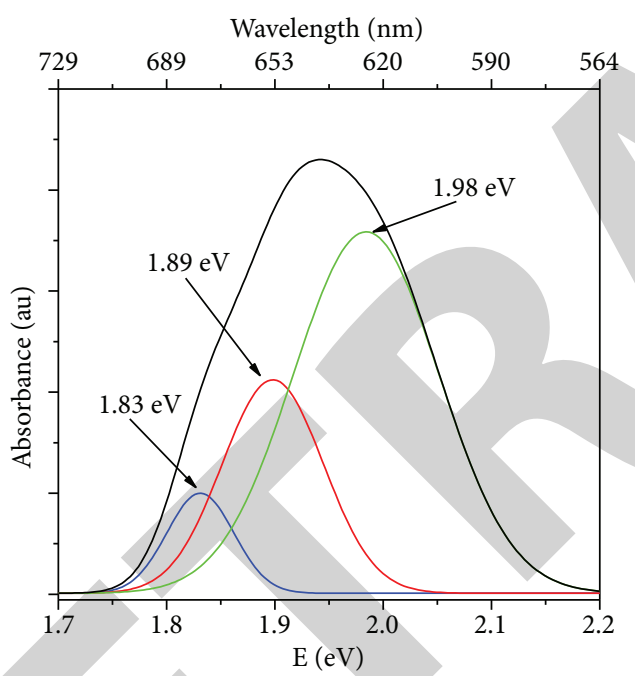

(c)

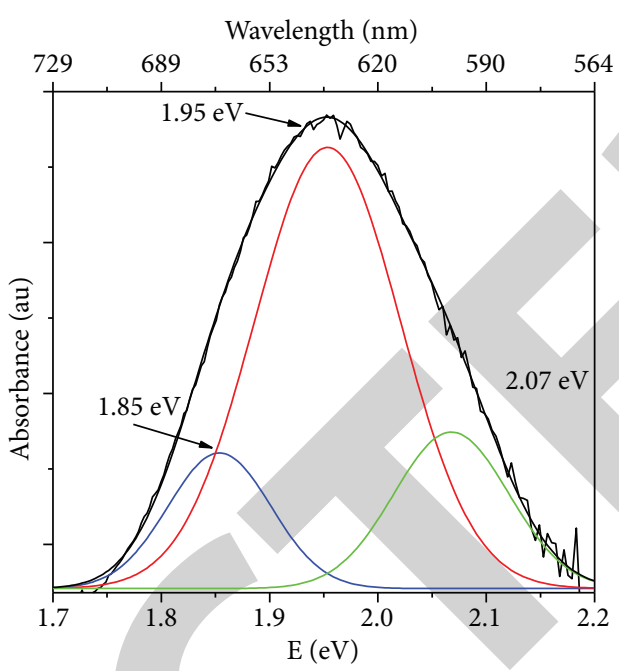

(b)

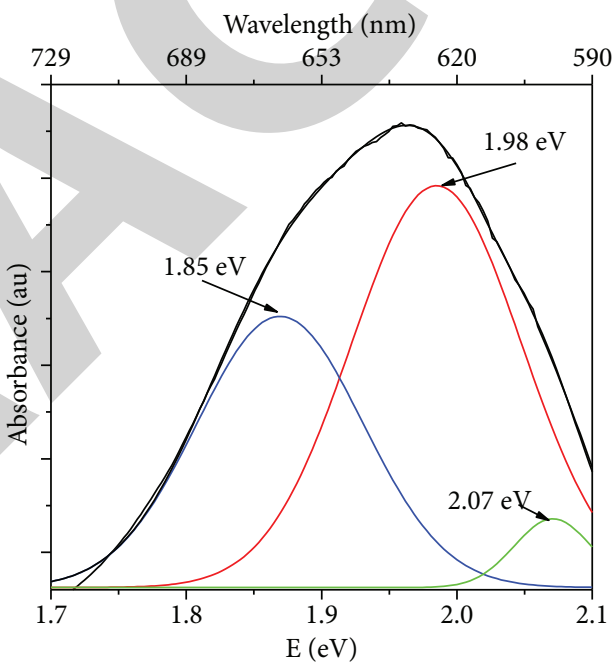

(d)

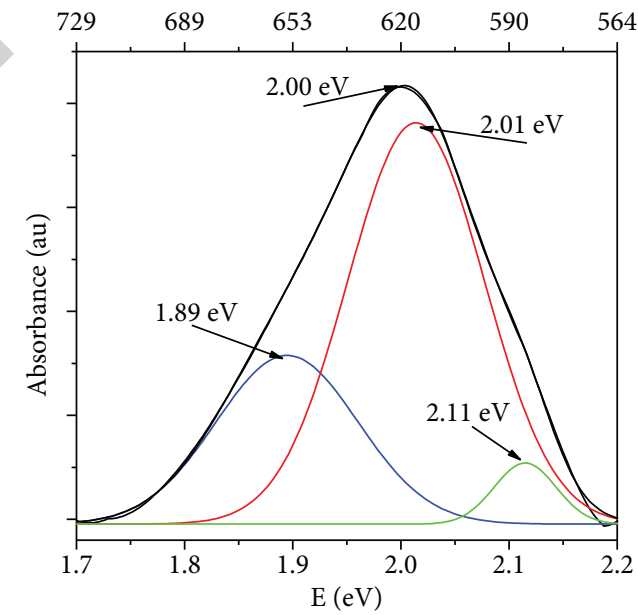

(e)

FIGURE 14: Deconvoluted absorbance versus photon energy spectra in the $1.7-2.2 \mathrm{eV}$ range for (a) PbS-PbS-T, (b) PbS-T5, (c) PbS-T10, and (d) PbS-T20, and (e) PdS-30 films. 


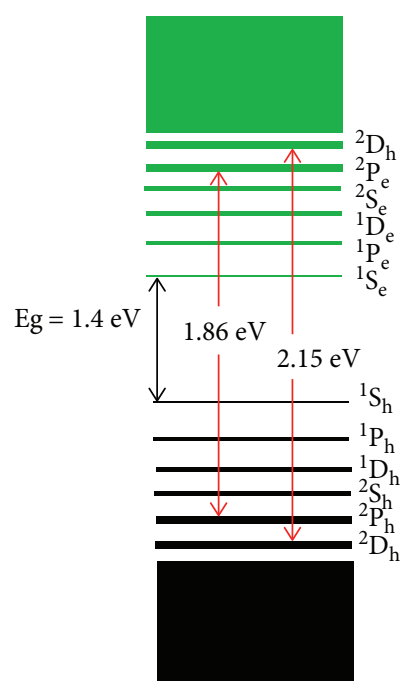

Figure 15: Quantized energy levels of PbS-PbS-T nanocrystals. The excitonic levels located at $\sim 1.4 \mathrm{eV}, \sim 1.8 \mathrm{eV}$, and $\sim 2.1 \mathrm{eV}$ are due to higher energy transitions from ${ }^{1} D_{\mathrm{h}} \rightarrow{ }^{1} D_{\mathrm{e}}$ and ${ }^{2} S_{\mathrm{h}} \rightarrow{ }^{2} S_{\mathrm{e}}$ states.

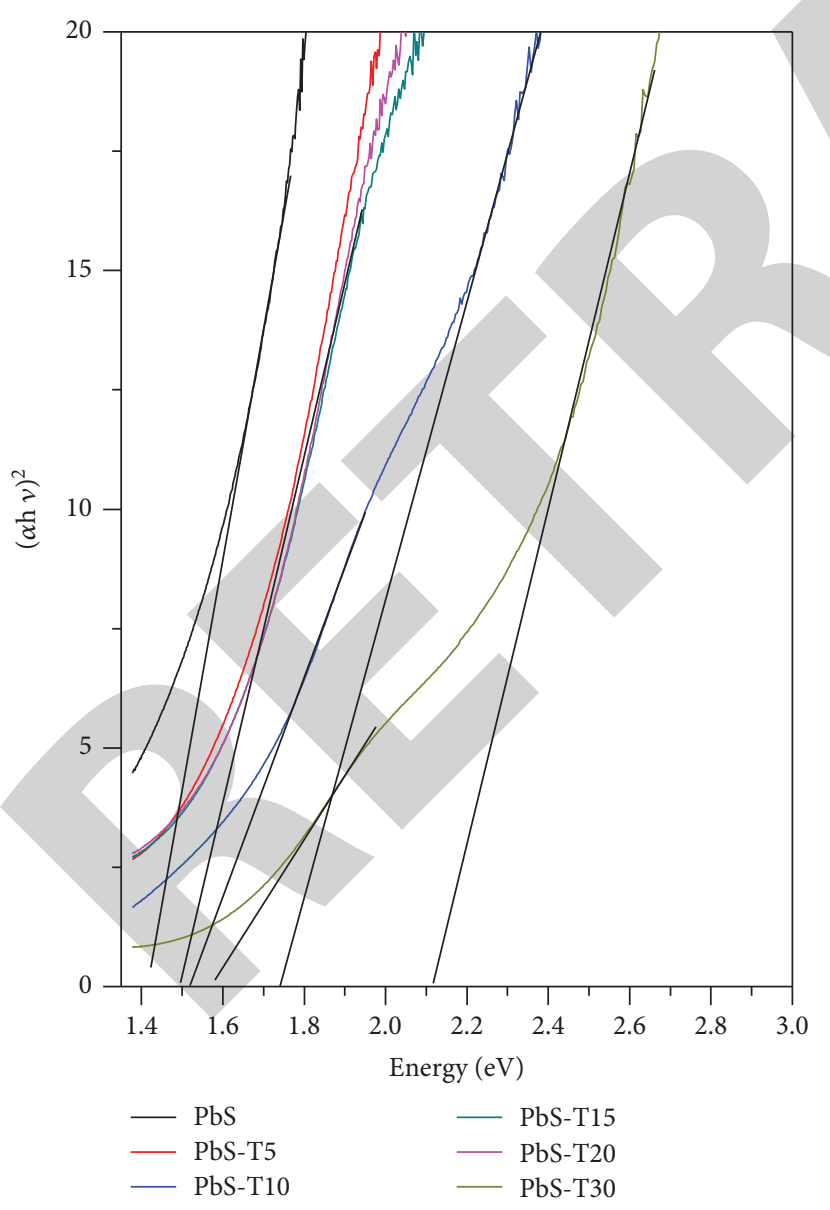

Figure 16: $(\alpha \mathrm{h} v)^{2}$ versus $\mathrm{h} v$ plot of PbS-PbS-T films, observing a $\mathrm{E}_{\mathrm{g}}$ shift associated with the PbS doping effect with the L-tryptophan biomolecule, thus presenting a quantum confinement effect.

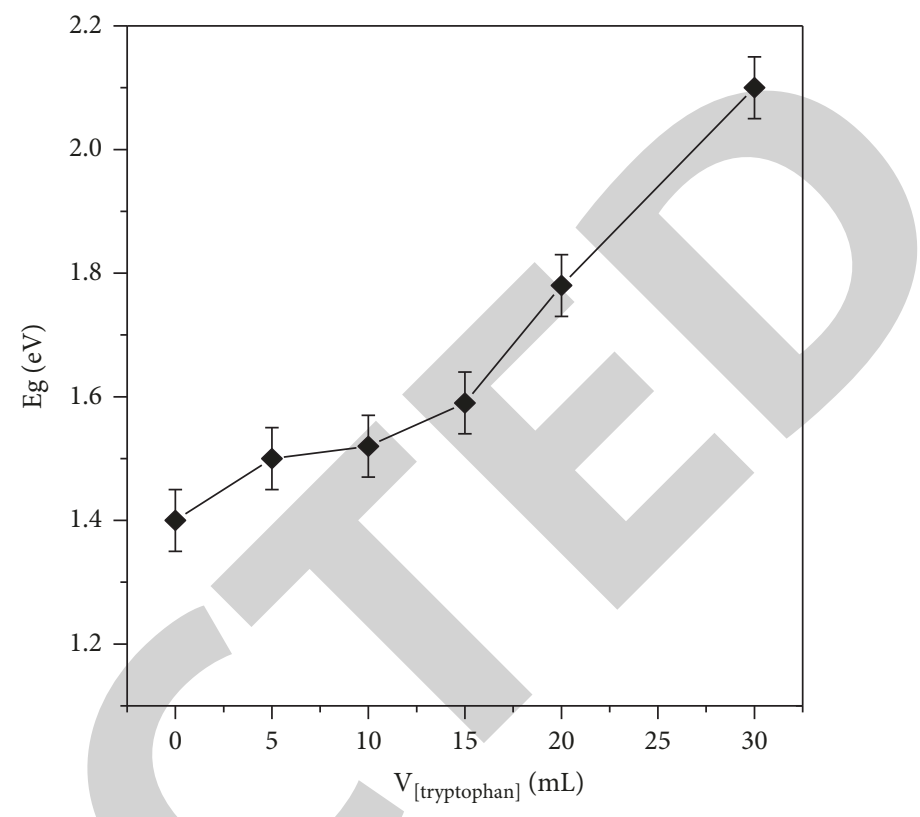

FIGURE 17: $\mathrm{E}_{\mathrm{g}}$ versus $\mathrm{V}_{\text {[L-tryptophan] }}$ plot of PbS-PbS-T nanocrystals. The behaviour of $\mathrm{E}_{\mathrm{g}}$ in this region displayed significant changes associated with the interaction of the L-tryptophan biomolecule.

TABLE 1: Experimental values of $\mathrm{E}_{\mathrm{g}}$ for PbS-PbS-T thin films.

\begin{tabular}{lc}
\hline Sample & $\mathrm{E}_{\mathrm{g}}(\mathrm{eV})$ \\
\hline $\mathrm{PbS}$ & 1.40 \\
$\mathrm{PbS}-\mathrm{T5}$ & 1.50 \\
$\mathrm{PbS}-\mathrm{T} 10$ & 1.52 \\
$\mathrm{PbS}-\mathrm{T} 15$ & 1.59 \\
$\mathrm{PbS}-\mathrm{T} 20$ & 1.78 \\
$\mathrm{PbS}-\mathrm{T} 30$ & 2.10 \\
\hline
\end{tabular}

biomolecule into the $\mathrm{PbS}$ matrix with, practically, no large damage to both the biomolecule and the nanocrystal matrix. Thus, it was found that the doping effect plays an important role in the formation of PbS nanocrystals. The optical and structural properties showed worth-noting changes, and the effect of strong quantum confinement observed in this semiconductor was due to the systematic doping with the Ltryptophan biomolecule. This method can probably be extended to doping with other organic molecules. We have thus developed a green and straightforward protocol to obtain $\mathrm{PbS}$ nanocrystals adjusted to match the ideal $\sim 1.5 \mathrm{eV}$ required for achieving a most efficient solar cell.

\section{Data Availability}

The .opj data used to support the findings of this study are available from the corresponding author upon request.

\section{Conflicts of Interest}

The authors declare that there is no conflict of interest regarding the publication of this paper. 


\section{Acknowledgments}

Thanks are due to Dr. J. R. Cerna and M. C. Noemi Melgoza Palma (Faculty of Ciencias Químicas-UAP), and Dr. R. Eguíbar Cuenca (VIEP-UAP) for their support. The authors gratefully recognize the financial support from VIEP of Benemérita Universidad Autónoma de Puebla (BUAP) and Vicerrectoría de Investigación y Estudios de Posgrado (VIEP-BUAP).

\section{References}

[1] S. Miri, A. Rostami, M. Dolatyari, H. Baghban, H. Rasooli, and E. Amini, "Fabrication of fast and sensitive IR-detectors based on $\mathrm{PbS}$ quantum dots passivated by organic ligands," Physica Status Solidi A: Applications and Materials Science, vol. 210, no. 2, pp. 420-424, 2013.

[2] M. A. Hines and G. D. Scholes, "Colloidal PbS nanocrystals with size-tunable near-infrared emission: observation of post-synthesis self-narrowing of the particle size distribution," Advanced Materials, vol. 15, no. 21, pp. 1844-1849, 2003.

[3] O. P. Moreno, R. G. Pérez, R. P. Merino, M. C. Portillo, G. H. Téllez, and E. R. Rosas, "Optical and structural properties of PbSIn $^{3}+$ nanocrystals grown by chemical bath," Thin Solid Films, vol. 616, pp. 800-807, 2016.

[4] M. C. Portillo, O. P. Moreno, R. G. Pérez et al., "Characterization and growth of doped-PbS in situ with $\mathrm{Bi}^{3+}, \mathrm{Cd}^{2+}$ and $\mathrm{Er}^{3+}$ ions by chemical bath," Materials Science in Semiconductor Processing, vol. 72, pp. 22-31, 2017.

[5] N. P. Dasgupta, W. Lee, and F. B. Prinz, "Atomic layer deposition of lead sulfide thin films for quantum confinement," Chemistry of Materials, vol. 21, no. 17, pp. 3973-3978, 2009.

[6] K. C. Preetha, K. V. Murali, A. J. Ragina, K. Deepa, and T. L. Remadevi, "Effect of cationic precursor $\mathrm{pH}$ on optical and transport properties of SILAR deposited nano crystalline PbS thin films," Current Applied Physics, vol. 12, no. 1, pp. 53-59, 2012.

[7] D. Liang, S. Tang, J. Liu, J. Liu, X. Lv, and L. Kang, "Large scale hydrothermal synthesis of PbS nanorods," Materials Letters, vol. 62 , no. 16, pp. 2426-2429, 2008.

[8] K. Bian, B. T. Richards, H. Yang et al., "Optical properties of $\mathrm{PbS}$ nanocrystal quantum dots at ambient and elevated pressure," Physical Chemistry Chemical Physics, vol. 16, no. 18, pp. 8515-8520, 2014.

[9] A. N. Chattarki, S. S. Kamble, and L. P. Deshmukh, "Role of $\mathrm{pH}$ in aqueous alkaline chemical bath deposition of lead sulfide thin films," Materials Letters, vol. 67, no. 1, pp. 39-41, 2012.

[10] O. Portillo-Moreno, R. Gutiérrez-Pérez, M. Chávez Portillo et al., "Growth of doped $\mathrm{PbS}: \mathrm{Co}^{2+}$ nanocrystals by chemical bath," Revista Mexicana de Física, vol. 62, pp. 456-460, 2016.

[11] W.-k. Koh, A. Y. Koposov, J. T. Stewart et al., "Heavily doped n-type $\mathrm{PbSe}$ and $\mathrm{PbS}$ nanocrystals using ground-state charge transfer from cobaltocene," Scientific Reports, vol. 3, no. 1, article 2004, 2013.

[12] S. A. Tomás, R. Lozada-Morales, O. Portillo, H. Lima-Lima, R. Palomino-Merino, and O. Zelaya, "Characterization of chemical bath deposited CdS thin films doped with methylene blue and $\mathrm{Er}^{3+}$," The European Physical Journal Special Topics, vol. 153, no. 1, pp. 299-302, 2008.
[13] B. Sahin, F. Bayansal, M. Yuksel, N. Biyikli, and H. A. Çetinkara, "Effect of coumarin concentration on the physical properties of $\mathrm{CdO}$ nanostructures," Ceramics International, vol. 40, no. 4, pp. 5237-5243, 2014.

[14] A. J. Bethune and N. A. S. Loud, Standard Aqueous Potential an Temperature Coefficients at $25^{\circ} \mathrm{C}$, C.C. Hampel, Skokie II, 1969.

[15] N. C. Polfer, J. Oomens, and R. C. Dunbar, "IRMPD spectroscopy of metal-ion/tryptophan complexes," Physical Chemistry Chemical Physics, vol. 8, no. 23, pp. 2744-2751, 2006.

[16] R. O. Priakumari, S. G. S. Sheba, and M. Gunasekaran, "Synthesis, growth and characterization of non-linear optical material: L-tryptophan p-nitrophenol (LTPNP) single crystal," Optik - International Journal for Light and Electron Optics, vol. 125, no. 17, pp. 4633-4636, 2014.

[17] X. Cao and G. Fischer, "Infrared spectral, structural, and conformational studies of zwitterionic L-tryptophan," The Journal of Physical Chemistry A, vol. 103, no. 48, pp. 9995-10003, 1999.

[18] S. Mohan, N. Puviarasan, and S. Bakkialakshmi, "Vibrational spectra and analysis of tryptophan," Asian Journal of Chemistry, vol. 11, no. 4, pp. 1137-1143, 1999.

[19] R. Gutiérrez Pérez, O. Portillo Moreno, M. Chávez Portillo et al., "Synthesis of $\mathrm{CdCO}_{3}$ in situ-doped- $\mathrm{Pb}^{2+}$ grown by chemical bath," Materials Letters, vol. 160, pp. 488-490, 2015.

[20] O. P. Moreno, F. J. M. Bustamante, M. C. Portillo et al., "Photoluminescent green emission band induced by the systematic change of -CH3, -OCH3 and naphthyl groups in chiral imines," Crystal Growth \& Design, vol. 18, no. 2, pp. 660-668, 2018.

[21] Z. Peralta-Inga, J. S. Murray, M. Edward Grice, S. Boyd, C. J. O'Connor, and P. Politzer, "Computational characterization of surfaces of model graphene systems," Journal of Molecular Structure: THEOCHEM, vol. 549, no. 1-2, pp. 147-158, 2001.

[22] P. K. Weiner, R. Langridge, J. M. Blaney, R. Schaefer, and P. A. Kollman, "Electrostatic potential molecular surfaces," Proceedings of the National Academy of Sciences of The United States of America, vol. 79, no. 12, pp. 3754-3758, 1982.

[23] M. Bedir, M. Öztaş, and M. Tekin, "The effect of the grain parameters on the characterization of polycrystalline CuS films," Arabian Journal for Science and Engineering, vol. 38, no. 7, pp. 1895-1902, 2013.

[24] W. C. Molenkamp, M. Watanabe, H. Miyata, and S. H. Tolbert, "Highly polarized luminescence from optical quality films of a semiconducting polymer aligned within oriented mesoporous silica," Journal of the American Chemical Society, vol. 126, no. 14, pp. 4476-4477, 2004.

[25] A. Fukuoka, H. Miyata, and K. Kuroda, "Alignment control of a cyanine dye using a mesoporous silica film with uniaxially aligned mesochannels," Chemical Communications, no. 2, pp. 284-285, 2003.

[26] A. Kariper, E. Güneri, F. Göde, C. Gümüş, and T. Özpozan, "The structural, electrical and optical properties of CdS thin films as a function of $\mathrm{pH}$," Materials Chemistry and Physics, vol. 129, no. 1-2, pp. 183-188, 2011.

[27] R. F. Chen, "Measurements of absolute values in biochemical fluorescence spectroscopy," Journal of Research of the National Bureau of Standards Section A: Physics and Chemistry, vol. 76A, no. 6, p. 593, 1972. 
[28] P. Papagiorgis, A. Stavrinadis, A. Othonos, G. Konstantatos, and G. Itskos, "The influence of doping on the optoelectronic properties of $\mathrm{PbS}$ colloidal quantum dot solids," Scientific Reports, vol. 6, no. 1, article 18735, 2016.

[29] Z. Q. Mamiyev and N. O. Balayeva, "Preparation and optical studies of PbS nanoparticles," Optical Materials, vol. 46, pp. 522-525, 2015.

[30] M. Salavati-Niasari, D. Ghanbari, and M. R. Loghman-Estarki, "Star-shaped PbS nanocrystals prepared by hydrothermal process in the presence of thioglycolic acid," Polyhedron, vol. 35, no. 1, pp. 149-153, 2012.

[31] F. D. Martinez-Mancera and J. L. Hernandez-Lopez, "Physical characterization and photoluminescence properties of thioglycolic acid-stabilized lead sulfide nanocrystals," Materials Chemistry and Physics, vol. 148, no. 3, pp. 1045-1054, 2014.

[32] G. Dukovic, F. Wang, D. Song, M. Y. Sfeir, T. F. Heinz, and L. E. Brus, "Structural dependence of excitonic optical transitions and band-gap energies in carbon nanotubes," Nano Letters, vol. 5, no. 11, pp. 2314-2318, 2005.

[33] J.-H. Chen, C.-G. Chao, J.-C. Ou, and T.-F. Liu, "Growth and characteristics of lead sulfide nanocrystals produced by the porous alumina membrane," Surface Science, vol. 601, no. 22, pp. 5142-5147, 2007.

[34] J. Zhang and X. Jiang, "Steady state photoinduced absorption of PbS quantum dots film," Applied Physics Letters, vol. 92, no. 14, pp. 141108-141103, 2008.

[35] M. Gao, Y. Yang, B. Yang, J. Shen, and X. Ai, "Effect of the surface chemical modification on the optical properties of polymer-stabilized PbS nanoparticles," Journal of the Chemical Society, Faraday Transactions, vol. 91, no. 22, pp. 4121-4125, 1995.

[36] A. A. Patel, F. Wu, J. Z. Zhang et al., "Synthesis, optical spectroscopy and ultrafast electron dynamics of $\mathrm{PbS}$ nanoparticles with different surface capping," The Journal of Physical Chemistry B, vol. 104, no. 49, pp. 11598-11605, 2000.

[37] A. Harriman, "Further comments on the redox potentials of tryptophan and tyrosine," The Journal of Physical Chemistry, vol. 91, no. 24, pp. 6102-6104, 1987.

[38] B.-R. Hyun, Y.-W. Zhong, A. C. Bartnik et al., "Electron injection from colloidal $\mathrm{PbS}$ quantum dots into titanium dioxide nanoparticles," ACS Nano, vol. 2, no. 11, pp. 2206-2212, 2008.

[39] W. P. Lim, H. Y. Low, and W. S. Chin, "IR-luminescent PbS - polystyrene nanocomposites prepared from random ionomers in solution," The Journal of Physical Chemistry B, vol. 108, no. 35, pp. 13093-13099, 2004.

[40] J. Xi, L. Feng, and L. Jiang, "A general approach for fabrication of superhydrophobic and superamphiphobic surfaces," Applied Physics Letters, vol. 92, no. 5, article 053102, 2008.

[41] A. S. Obaid, M. A. Mahdi, Z. Hassan, and M. Bououdina, "Characterization of nanocrystalline $\mathrm{PbS}$ thin films prepared using microwave-assisted chemical bath deposition," Materials Science in Semiconductor Processing, vol. 15, no. 5, pp. 564-571, 2012.

[42] R. Das and R. Kumar, "Preparation of nanocrystalline PbS thin films and effect of Sn doping and annealing on their structural and optical properties," Materials Research Bulletin, vol. 47, no. 2, pp. 239-246, 2012.
[43] J. Chang and E. R. Waclawik, "Colloidal semiconductor nanocrystals: controlled synthesis and surface chemistry in organic media," RSC Advances, vol. 4, no. 45, pp. 23505-23527, 2014.

[44] A. Pimachev and Y. Dahnovsky, "Optical and magnetic properties of PbS nanocrystals doped by manganese impurities," The Journal of Physical Chemistry C, vol. 119, no. 29, pp. 16941-16946, 2015.

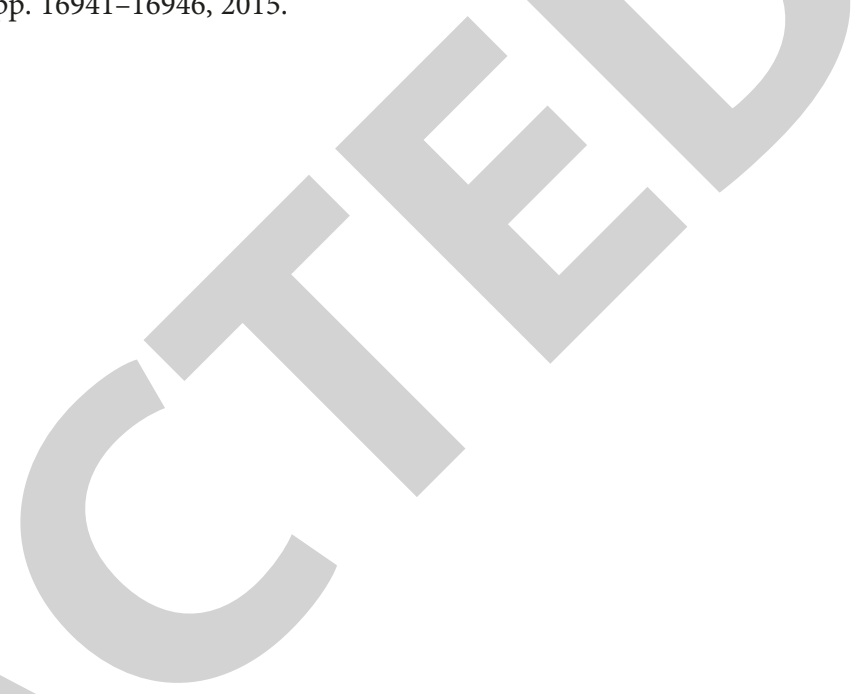

